\title{
Jacobi-like algorithms for the indefinite generalized Hermitian eigenvalue problem
}

\author{
Christian Mehl*
}

\begin{abstract}
We discuss structure-preserving Jacobi-like algorithms for the solution of the indefinite generalized Hermitian eigenvalue problem. We discuss a method based on the solution of Hermitian 4-by-4 subproblems which generalizes the Jacobi-like method of Bunse-Gerstner/Faßbender for Hamiltonian matrices. Furthermore, we discuss structure-preserving Jacobi-like methods based on the solution of non-Hermitian 2by-2 subproblems. For these methods a local convergence proof is given. Numerical test results for the comparison of the proposed methods are presented.
\end{abstract}

Keywords. Jacobi-like method, Hermitian pencil, eigenvalues

AMS subject classifications. $65 \mathrm{~F} 15$

\section{Introduction}

The generalized Hermitian eigenvalue problem arises in many applications. One important example is the linear quadratic optimal control problem, see $[18,19,24]$ and the references therein. This is the problem of minimizing the cost functional

$$
\frac{1}{2} \int_{t_{0}}^{\infty}\left(x(t)^{*} Q x(t)+u(t)^{*} R u(t)+u(t)^{*} S^{*} x(t)+x(t)^{*} S u(t)\right) d t
$$

subject to the dynamics

$$
\begin{aligned}
E \dot{x}(t) & =A x(t)+B u(t), \quad t_{0}<t \\
x\left(t_{0}\right) & =x_{0},
\end{aligned}
$$

where $A, E, Q \in \mathbb{C}^{n \times n}, B, S \in \mathbb{C}^{n \times m}, R \in \mathbb{C}^{m \times m}, Q, R$ Hermitian, $x_{0}, x(t), u(t) \in \mathbb{C}^{n}$, and $t_{0}, t \in \mathbb{R}$. It is known that solutions of (1)-(3) can be obtained via the solution of a

${ }^{*}$ Technische Universität Berlin, Fakultät II; Institut für Mathematik, D-10623 Berlin, Germany; mehl@math. tu-berlin.de 
boundary value problem, see $[23,24]$ and the references therein. For the solution of this boundary value problem one has to compute deflating subspaces of the matrix pencil

$$
\lambda \mathcal{A}-\mathcal{B}=\lambda\left[\begin{array}{ccc}
0 & -E^{*} & 0 \\
E & 0 & 0 \\
0 & 0 & 0
\end{array}\right]-\left[\begin{array}{ccc}
Q & A^{*} & S \\
A & 0 & B \\
S^{*} & B^{*} & R
\end{array}\right] .
$$

Setting $\mathcal{G}_{0}=i \mathcal{A}$ and $\mathcal{H}_{0}=\mathcal{B}$, we find that $\lambda \mathcal{G}_{0}-\mathcal{H}_{0}$ is a Hermitian pencil, i.e., both $\mathcal{G}_{0}$ and $\mathcal{H}_{0}$ are Hermitian. Clearly, both pencils $\lambda \mathcal{A}-\mathcal{B}$ and $\lambda \mathcal{G}_{0}-\mathcal{H}_{0}$ have the same right deflating subspaces and the eigenvalues of $\lambda \mathcal{G}_{0}-\mathcal{H}_{0}$ coincide with the eigenvalues of $\lambda \mathcal{A}-\mathcal{B}$ multiplied by $i$.

Another important application is the Hermitian quadratic eigenvalue problem, i.e., the problem of finding $\lambda \in \mathbb{C}$ and $x \in \mathbb{C}^{n} \backslash\{0\}$ such that $\left(\lambda^{2} M+\lambda C+K\right) x=0$, where $M, C, K \in \mathbb{C}^{n \times n}$ are Hermitian. This problem arises for example in the analysis of geometrical nonlinear buckling structures with finite element methods (see $[1,12]$ ) or in the theory of damped oscillatory systems (see [9, 17]). With the substitution $\mu=\frac{1}{\lambda}$ for $\lambda \neq 0$, the problem can be linearized such that it reduces to the generalized Hermitian eigenvalue problem

$$
\mu\left[\begin{array}{cc}
C & M \\
M & 0
\end{array}\right]\left[\begin{array}{c}
\lambda x \\
x
\end{array}\right]=\left[\begin{array}{cc}
-K & 0 \\
0 & M
\end{array}\right]\left[\begin{array}{c}
\lambda x \\
x
\end{array}\right]
$$

see, e.g., [12].

There are numerous algorithms for the solution of the generalized Hermitian eigenvalue problem $\lambda \mathcal{G} x=\mathcal{H} x$ for the case that $\mathcal{G}$ (or $\mathcal{H}$, respectively) is positive definite. For example, one could compute the Cholesky factorization $\mathcal{G}=L L^{T}$ and then consider the standard eigenvalue problem $\lambda I-L^{-1} A L^{-T}$, see [11] and the references therein. But, there is no software available that takes advantage of the symmetry and possible spectral properties for the case that the generalized eigenvalue problem $\lambda \mathcal{G}-\mathcal{H}$ is indefinite [32], although these problems arise frequently in applications. Indeed, easy examples show that the eigenvalue problems $\lambda \mathcal{G}_{0} x=\mathcal{H}_{0} x$ and (4) are in general indefinite.

On the other hand, it is well known that the indefinite generalized Hermitian eigenvalue problem is related to the standard eigenvalue problem for a matrix that is selfadjoint with respect to an indefinite inner product. Indeed, if $\mathcal{G}$ is nonsingular, then $\lambda \mathcal{G}-\mathcal{H}$ is equivalent to the pencil $\lambda I-\mathcal{G}^{-1} \mathcal{H}$, where $\mathcal{G}^{-1} \mathcal{H}$ is selfadjoint with respect to the indefinite inner product induced by $\mathcal{G}$, i.e., $\left(\mathcal{G}^{-1} \mathcal{H}\right)^{*} \mathcal{G}=\mathcal{G}\left(\mathcal{G}^{-1} \mathcal{H}\right)$. This fact gives rise to the following basic idea: given a method for the solution of the standard eigenvalue problem for a matrix that is structured with respect to an indefinite inner product, try to generalize this method to Hermitian pencils.

In recent years, there has been interest in generalizing Jacobi's algorithm for the symmetric eigenvalue problem to other structured eigenvalue problems, in particular to Hamiltonian or doubly structured eigenvalue problems, see $[2,3,8]$. Hamiltonian matrices are skew-adjoint with respect to the indefinite inner product induced by the matrix

$$
J:=J_{n}=\left[\begin{array}{cc}
0 & I_{n} \\
-I_{n} & 0
\end{array}\right] \in \mathbb{C}^{2 n \times 2 n}
$$


where $I_{n}$ denotes the $n \times n$ identity matrix. Thus, a matrix $H \in \mathbb{C}^{2 n \times 2 n}$ is Hamiltonian if and only if $H J+J H^{*}=0$. Analogously, a matrix $S \in \mathbb{C}^{2 n \times 2 n}$ is called skew-Hamiltonian if and only if $S J-J S^{*}=0$. Following the basic idea just mentioned, it will be shown in this paper that Jacobi-like methods for Hamiltonian matrices can be generalized to the case of Hermitian pencils. We will focus on even-sized pencils. A generalization to oddsized Hermitian pencils is possible, but this needs a more detailed discussion which is not presented here.

The interest in generalized Jacobi-like methods is due to several reasons. First, these methods are inherently parallelizable and backward stable if one restricts oneself to unitary transformation matrices. (E.g., backward stability for the Jacobi-like methods proposed in [8] has been shown in [31].) Moreover, Jacobi's classical algorithm is more accurate than the $Q R$-algorithm if a proper stopping criterion is used $[5,20]$ and has the advantage of converging very fast, if the matrix under consideration is already close to being diagonal. Thus, given a structured eigenvalue problem with a matrix already close to a condensed form that is to be computed, Jacobi-like algorithms are expected to converge much faster than methods that ignore this special property. Hence, Jacobi-like methods may be attractive for the solution of eigenvalue problems that depend on a parameter, e.g., $H_{\infty}$ control problems [34], where one has to compute the eigenvalues of a Hamiltonian matrix $H(\gamma)$ depending continuously on a real parameter $\gamma$. Once, a Hamiltonian Schur form for a matrix $H\left(\gamma_{0}\right)$ with some specific value $\gamma_{0}$ has been computed, the corresponding transformations will transform matrices $H(\gamma)$, where $\gamma$ is sufficiently close to $\gamma_{0}$, to a form close to Hamiltonian Schur form. It is then reasonable to use a Jacobi-like method for the solution of the eigenvalue problem with $H(\gamma)$.

The paper is organized as follows. After reviewing anti-triangular forms for Hermitian pencils and relating them to the Hamiltonian Schur form of Hamiltonian matrices in Section 2, we will introduce the algorithm JIGH4 (a Jacobi-like method that is based on the solution of $4 \times 4$ subproblems and that generalizes the algorithm of Bunse-Gerstner/Faßbender for Hamiltonian matrices [2]) in Section 3. In Section 4, we will propose the algorithm JIGH2 (a method based on the solution of $2 \times 2$ subproblems) that we will prove to be locally quadratically convergent in Section 5. Since this algorithm may sometimes stagnate, we propose a slightly modified version called MJIGH2 in Section 6 that is also locally quadratically convergent, but that does not stagnate in practice. In Section 7, we present numerical test results for the comparison of the methods JIGH4 and MJIGH2.

\section{Anti-triangular forms for Hermitian pencils}

The Jacobi-like algorithms that will be presented in this paper are supposed to be structure preserving algorithms. (The eigenvalues of Hermitian pencils occur in pairs $(\lambda, \bar{\lambda})$, see, e.g., [30], and we want to maintain this property.) The structure of Hermitian pencils is preserved under congruence transformations $\lambda \mathcal{G}-\mathcal{H} \mapsto P^{*}(\lambda \mathcal{G}-\mathcal{H}) P$, where $P$ is nonsingular, and since we are interested in restricting ourselves to unitary transformations for the sake of numerical stability, we will consider the problem of finding condensed forms 
for Hermitian pencils under simultaneous unitary similarity. The classical Schur form would be such that both $\mathcal{G}$ and $\mathcal{H}$ are diagonal. However, it is well known that a pair of Hermitian matrices is simultaneously unitarily diagonalizable if and only if the matrices commute. On the other hand, indefinite Hermitian pencils may have complex conjugate eigenvalues and in this case a diagonal form cannot exist, even if we allow general congruence transformations instead of unitary ones. An alternative to diagonal forms are the so-called anti-triangular forms that have been introduced in [22]. An $n \times n$-matrix $A=\left(a_{j k}\right)$ is called lower anti-triangular if $a_{j k}=0$ for all $j, k$ such that $j+k \leq n$, i.e., if $A$ has the pattern

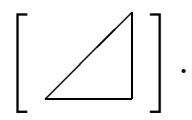

Definition 1 We say that a Hermitian pencil $\lambda \mathcal{G}-\mathcal{H} \in \mathbb{C}^{n \times n}$ is in anti-triangular form if both $\mathcal{G}$ and $\mathcal{H}$ are lower anti-triangular.

Clearly, anti-triangular forms display the eigenvalues of the pencil and a nested set of deflating subspaces. Moreover, anti-triangular forms for Hermitian pencils are related to Schur-like forms for skew-Hamiltonian/Hamiltonian pencils that have been discussed in [21], and thus, they are related to Hamiltonian Schur forms for Hamiltonian matrices. (A skew-Hamiltonian/Hamiltonian pencil is a pencil $\lambda S-H$ such that $S$ is skew-Hamiltonian and $H$ is Hamiltonian.) If $\lambda S-H$ is a skew-Hamiltonian/Hamiltonian pencil in Schur-like form, i.e., $\lambda S-H$ has the pattern

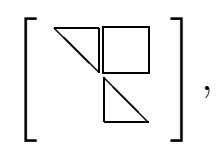

then $\lambda i J S-J H$ is a Hermitian pencil and has the pattern

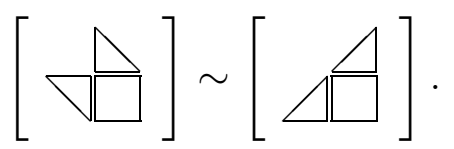

Here, $\sim$ denotes congruence, i.e., $\lambda i J S-J H$ is congruent to a pencil in anti-triangular form. From this point of view, anti-triangular forms are the natural forms to look for if one is interested in obtaining condensed forms for indefinite Hermitian pencils under unitary transformations.

It is well known that the Hamiltonian Schur form for Hamiltonian matrices or analogously the Schur-like form for skew-Hamiltonian/Hamiltonian pencils does not always exist if the matrix or pencil under consideration has purely imaginary eigenvalues. A similar observation can be made for anti-triangular forms for Hermitian pencils. Here, real eigenvalues are the ones that might cause problems. A necessary and sufficient condition for the existence of anti-triangular forms for Hermitian pencils was obtained in [22] Theorem 15 and Corollary 21. We will not quote those results in full generality, but only the following important special case:

Proposition 2 Let $\lambda \mathcal{G}-\mathcal{H} \in \mathbb{C}^{n \times n}$ be a Hermitian pencil having no real eigenvalues if $n$ is even, or exactly one real eigenvalue (counting multiplicities) if $n$ is odd. Then there exists a unitary matrix $\mathcal{U} \in \mathbb{C}^{n \times n}$ such that $\lambda \mathcal{U}^{*} \mathcal{G U}-\mathcal{U}^{*} \mathcal{H U}$ is in anti-triangular form. 


\section{A method working on 4-by-4 subproblems (JIGH4)}

In this section, we will generalize the Jacobi-like algorithm for Hamiltonian matrices by Bunse-Gerstner/Faßbender [2] to the case Hermitian pencils. We start with the following short survey.

\subsection{A short survey on Jacobi methods}

The idea of Jacobi's original method [15] for the diagonalization of a symmetric matrix $A=\left(a_{i j}\right) \in \mathbb{R}^{n \times n}$ is to successively apply similarity transformations with rotation matrices such that each one diagonalizes a particular $2 \times 2$ submatrix of $A$. In each step, one chooses a pivot pair of indices $(k, l)$ and applies a similarity transformation with a rotation matrix $U=\left(u_{i j}\right)$ that annihilates the entries $(l, k)$ and $(k, l)$ of $A$. Here, $U$ coincides with the identity except for the elements $u_{k k}=u_{l l}=\cos \alpha$ and $-u_{k l}=u_{l k}=\sin \alpha$.

The classical Jacobi algorithm chooses indices $(k, l)$ such that $a_{k l}^{2}$ is maximal in each step while cyclic Jacobi methods use fixed sequences of indices $(k, l)$ such as

$$
(k, l)=(1,2),(1,3), \ldots,(1, n),(2,3), \ldots,(2, n), \ldots,(n-1, n),
$$

where every possible index pair is considered exactly once. Usually, the performance of $n(n-1) / 2$ Jacobi steps is called a sweep, and hence, cyclic Jacobi methods consider every possible index pair once in a sweep. Under certain conditions both the classical Jacobi method and the cyclic Jacobi methods are convergent and their asymptotic convergence rate is quadratic, see $[14,16,26,33]$.

Jacobi's method has been adapted to other classes of matrices, see, e.g., [2, 3, 6, 8, 10, 13, 25]. In particular, Stewart [28] and Eberlein [7] generalized Jacobi's method to the computation of the Schur decomposition of a general complex matrix. Similarly to Jacobi's original method, a pivot pair $(k, l)$ is chosen and a similarity transformation with a rotation $U=\left(u_{i j}\right)$ is applied that yields the Schur decompositions of the corresponding $2 \times 2$ subproblem. Again, $U$ coincides with the identity except for the elements $u_{k k}=u_{l l}=$ $\cos \alpha, u_{k l}=-e^{-i \theta} \sin \alpha$, and $u_{l k}=e^{i \theta} \sin \alpha$. While Stewart proposed to only use pivot elements from the lower subdiagonal, Eberlein proposed to allow all elements from the lower triangular part of the matrix as pivot elements. Moreover, both methods differ in the choice of rotation parameters. Charlier and van Dooren [4] generalized Stewart's method to the computation of the generalized Schur decomposition of matrix pencils and were able to prove global convergence under certain restrictions, whereas there is no convergence proof for the method of Eberlein so far. But, it has been observed that Eberlein's method converges faster than Stewart's method if the matrix under consideration is not close to being normal.

In 1990, Byers [3] adapted Stewart's method to the computation of the Hamiltonian Schur form of a Hamiltonian matrix $A$. In order to preserve the structure of Hamiltonian matrices in each step, the proposed algorithm considers $4 \times 4$ subproblems rather than $2 \times 2$ subproblems. However, it has been observed that convergence may be very slow if 
the matrix under consideration is not close to a normal matrix. Sometimes, the method does not converge at all. One problem is that some of the $4 \times 4$ subproblems may have purely imaginary eigenvalues such that a reduction as proposed in the algorithm is not possible. Subproblems with purely imaginary eigenvalues may occur even when the original matrix $A$ has no purely imaginary eigenvalues.

In 1997, Bunse-Gerstner/Faßbender proposed a different Jacobi-like algorithm that generalizes Eberlein's method. Again, the algorithm considers $4 \times 4$ subproblems instead of $2 \times 2$ subproblems. As for Byers' method, subproblems with purely imaginary eigenvalues may occur such that a reduction in the current step is not necessarily possible. However, the authors were able to observe convergence for all their test problems and found that the performance of their method was superior to the one of Byers' when the Hamiltonian matrix under consideration was not normal. Unfortunately, there is no convergence proof for this method so far.

It is the aim of this section to generalize Jacobi-like methods for Hamiltonian matrices to the case of indefinite Hermitian pencils. Given the fact that the convergence behavior of Bunse-Gerstner/Faßbender's method is superior to the one of Byers' method if the matrix under consideration is not normal (a pair of simultaneously unitarily anti-diagonalizable Hermitian matrices could be interpreted as a Hermitian pencil corresponding to a normal Hamiltonian matrix), we will focus on the first method. Moreover, we will restrict ourselves to the case of even-sized pencils. Generalizations to the case of odd-sized pencils are possible but will involve the solution of some $3 \times 3$ subproblems. Details will not be presented in this paper.

\subsection{Solving the eigenvalue problem for 4-by-4 Hermitian pencils}

The anti-triangular form for $4 \times 4$ Hermitian pencils (provided that this form exists) can be computed by adapting the method for the computation of Schur-like forms for skewHamiltonian/Hamiltonian pencils developed in [21]. This requires an a-priori knowledge of eigenvalues and eigenvectors. (In our MATLAB implementation, the routine 'eig' has been used for the solution of the $4 \times 4$ eigenvalue problems.) If $\lambda \mathcal{G}-\mathcal{H}$ is a regular $4 \times 4$ Hermitian pencil we have to distinguish three different cases:

(i) $\lambda \mathcal{G}-\mathcal{H}$ has two pairs of complex conjugate eigenvalues;

(ii) $\lambda \mathcal{G}-\mathcal{H}$ has two real eigenvalues and a pair of complex conjugate eigenvalues;

(iii) $\lambda \mathcal{G}-\mathcal{H}$ has four real eigenvalues.

Here, possible infinite eigenvalues are considered to be real eigenvalues. If we are in Case (i) or (ii), let $v$ be an eigenvector associated with a nonreal eigenvalue $\lambda_{1}$ of $\lambda \mathcal{G}-\mathcal{H}$. Then $\mathcal{G} v($ or $\mathcal{H} v$ ) and $v$ are orthogonal, because of

$$
\lambda_{1} v^{*} \mathcal{G} v=v^{*} \mathcal{H} v=\left(v^{*} \mathcal{H} v\right)^{*}=\bar{\lambda}_{1} v^{*} \mathcal{G} v .
$$


Thus, there exists a unitary matrix $Q=[q 1, q 2, q 3, q 4]$ such that $q 1=v /\|v\|$ and $q 4=$ $\mathcal{G} v /\|\mathcal{G} v\|$. We then obtain

$$
Q^{*}(\lambda \mathcal{G}-\mathcal{H}) Q=\lambda\left[\begin{array}{cccc}
0 & 0 & 0 & g_{14} \\
0 & g_{22} & g_{23} & g_{24} \\
0 & \bar{g}_{23} & g_{33} & g_{34} \\
\bar{g}_{14} & \bar{g}_{24} & \bar{g}_{34} & g_{44}
\end{array}\right]-\left[\begin{array}{cccc}
0 & 0 & 0 & h_{14} \\
0 & h_{22} & h_{23} & h_{24} \\
0 & \bar{h}_{23} & h_{33} & h_{34} \\
\bar{h}_{14} & \bar{h}_{24} & \bar{h}_{34} & h_{44}
\end{array}\right]
$$

The eigenvalues of this pencil are $\lambda_{1}=\bar{h}_{14} / \bar{g}_{14}$ and $\bar{\lambda}_{1}$ together with the eigenvalues of the subpencil

$$
\lambda\left[\begin{array}{ll}
g_{22} & g_{23} \\
\bar{g}_{23} & g_{33}
\end{array}\right]-\left[\begin{array}{ll}
h_{22} & h_{23} \\
\bar{h}_{23} & h_{33}
\end{array}\right] .
$$

If we are in Case (ii), then (7) has two real eigenvalues and we stop the reduction process. The form (6) will be called partial anti-triangular form. If we are in Case (i), then (7) has a pair of complex conjugate eigenvalues and we repeat the procedure above to transform this subpencil to anti-triangular form. For our $4 \times 4$ pencil, this means that there exists a unitary matrix $\tilde{Q}$ such that

$$
\tilde{Q}^{*}(\lambda \mathcal{G}-\mathcal{H}) \tilde{Q}=\lambda\left[\begin{array}{cccc}
0 & 0 & 0 & g_{14} \\
0 & 0 & \tilde{g}_{23} & \tilde{g}_{24} \\
0 & \overline{\tilde{g}}_{23} & \tilde{g}_{33} & \tilde{g}_{34} \\
\bar{g}_{14} & \overline{\tilde{g}}_{24} & \overline{\tilde{g}}_{34} & g_{44}
\end{array}\right]-\left[\begin{array}{cccc}
0 & 0 & 0 & h_{14} \\
0 & 0 & \tilde{h}_{23} & \tilde{h}_{24} \\
0 & \overline{\tilde{h}}_{23} & \tilde{h}_{33} & \tilde{h}_{34} \\
\bar{h}_{14} & \overline{\tilde{h}}_{24} & \tilde{\tilde{h}}_{34} & h_{44}
\end{array}\right]
$$

Clearly, there is a continuum of simultaneous unitary similarity transformations that bring $\lambda \mathcal{G}-\mathcal{H}$ to anti-triangular form, if there are no real eigenvalues. Thus, the question arises, which one to choose, in order to guarantee best convergence properties of the corresponding Jacobi-like method. One could choose the matrix $\tilde{Q}$ that is nearest to the identity or such that the sum of the absolute values of the left lower triangular part of $\tilde{Q}$ is minimized. At this stage, we do not know which strategy is the best. The two strategies mentioned above work well for pencils that are already close to anti-triangular form, but they seem to slow down the convergence process for random Hermitian pencils. In our numerical experiments, we observed fastest convergence by using the following strategy for choosing the eigenvectors used in the reduction procedure for Case (i) and Case (ii) above:

1. for the first reduction step (towards partial anti-triangular form), consider only eigenvectors associated with eigenvalues with negative imaginary part and among those choose the normalized eigenvector that is closest to the first unit vector;

2. for the second reduction step (only in Case (i)), choose the eigenvector associated with the eigenvalue with negative imaginary part.

Thus, we only consider eigenvectors associated with eigenvalues with negative imaginary parts. (Clearly, one can also consider an analogous strategy that only chooses eigenvectors associated with eigenvalues with positive imaginary part.) 


\subsection{The algorithm JIGH4}

We have provided all ingredients to formulate the algorithm JIGH4 (Jacobi-like algorithm for the indefinite generalized Hermitian eigenvalue problem based on $\underline{4} \times 4$ subproblems). We use a cyclic-by-row-type ordering scheme of pivot indices, e.g., in the $8 \times 8$ case:

$$
(1,2,7,8),(1,3,6,8),(1,4,5,8),(2,3,6,7),(2,4,5,7),(3,4,5,6),(1,2,7,8), \ldots
$$

Throughout the rest of this paper, if $X=\left(x_{i j}\right) \in \mathbb{C}^{2 n \times 2 n}$, let $X_{k l}^{(4)}$ denote the matrix

$$
X_{k l}^{(4)}=\left[\begin{array}{cccc}
x_{k k} & x_{k l} & x_{k, 2 n+1-l} & x_{k, 2 n+1-k} \\
x_{l k} & x_{l l} & x_{l, 2 n+1-l} & x_{l, 2 n+1-k} \\
x_{2 n+1-l, k} & x_{2 n+1-l, l} & x_{2 n+1-l, 2 n+1-l} & x_{2 n+1-l, 2 n+1-k} \\
x_{2 n+1-k, k} & x_{2 n+1-k, l} & x_{2 n+1-k, 2 n+1-l} & x_{2 n+1-k, 2 n+1-k}
\end{array}\right] .
$$

Then algorithm JIGH4 takes the form as given below.

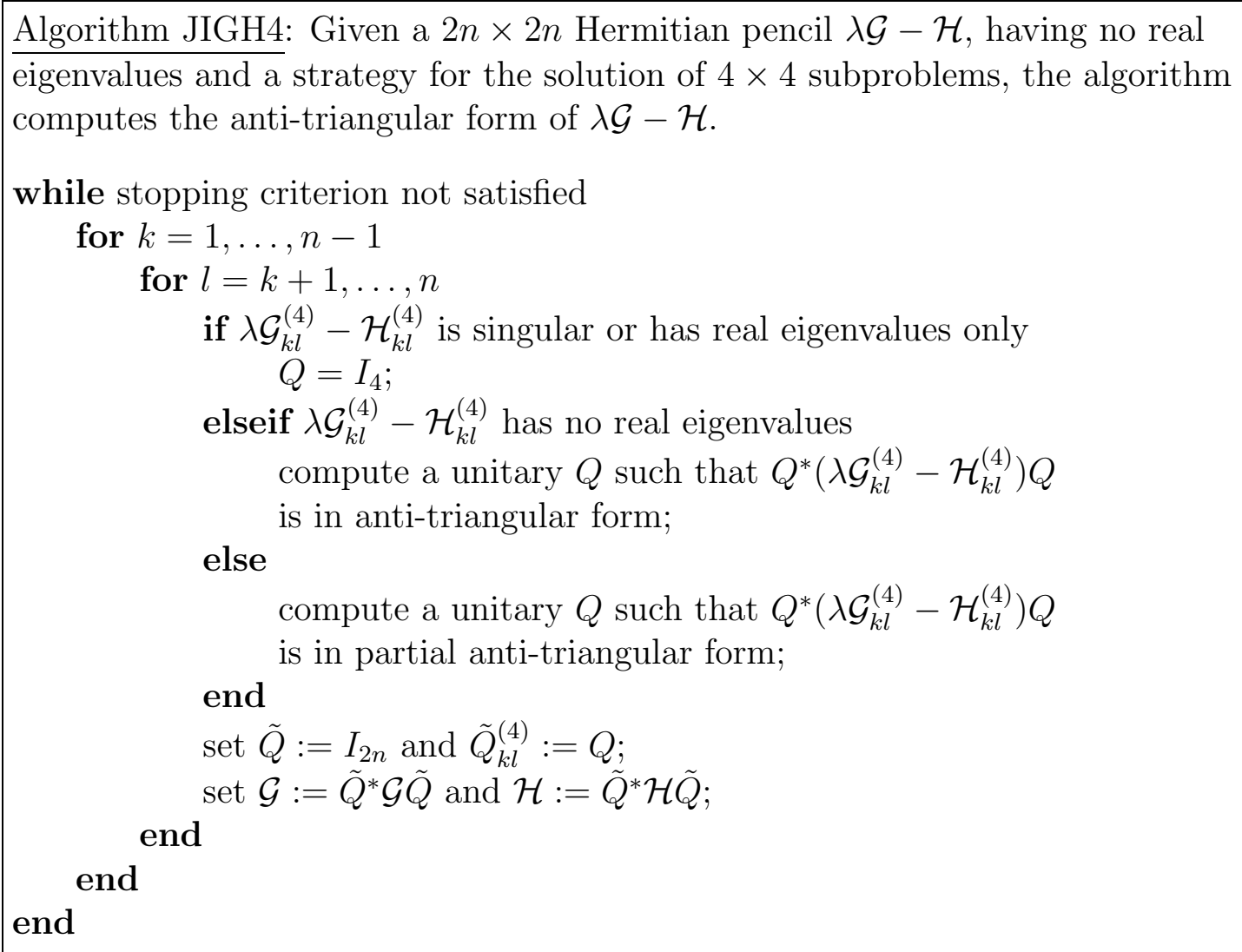

Following [2], a 'sweep' denotes one performance of the 'while'-loop, although this does not correspond to $n(n-1) / 2$ single Jacobi-steps. Thus, assuming that all occurring $4 \times 4$ subproblems can be solved, a sweep in the $8 \times 8$ case proceeds as indicated in the sketch below. The current subpencil is marked by the bold and the blank discs, where the blank discs display the elements that will be annihilated in the current step. 


$$
\begin{aligned}
& {\left[\begin{array}{llllllll}
0 & 0 & \cdot & \cdot & \cdot & \cdot & 0 & \bullet \\
0 & 0 & \cdot & \cdot & \cdot & \cdot & \bullet & \bullet \\
\cdot & \cdot & \cdot & \cdot & \cdot & \cdot & \cdot & \cdot \\
\cdot & \cdot & \cdot & \cdot & \cdot & \cdot & \cdot & \cdot \\
\cdot & \cdot & \cdot & \cdot & \cdot & \cdot & \cdot & \cdot \\
\cdot & \cdot & \cdot & \cdot & \cdot & \cdot & \cdot & \cdot \\
0 & \bullet & \cdot & \cdot & \cdot & \cdot & \bullet & \bullet \\
\bullet & \bullet & \cdot & \cdot & \cdot & \cdot & \bullet & \bullet
\end{array}\right] \rightsquigarrow\left[\begin{array}{cccccccc}
0 & \cdot & 0 & \cdot & \cdot & 0 & \cdot & \bullet \\
\cdot & \cdot & \cdot & \cdot & \cdot & \cdot & \cdot & \cdot \\
0 & \cdot & 0 & \cdot & \cdot & \bullet & \cdot & \bullet \\
\cdot & \cdot & \cdot & \cdot & \cdot & \cdot & \cdot & \cdot \\
\cdot & \cdot & \cdot & \cdot & \cdot & \cdot & \cdot & \cdot \\
0 & \cdot & \bullet & \cdot & \cdot & \bullet & \cdot & \bullet \\
\cdot & \cdot & \cdot & \cdot & \cdot & \cdot & \cdot & \cdot \\
\bullet & \cdot & \bullet & \cdot & \cdot & \bullet & \cdot & \bullet
\end{array}\right] \rightsquigarrow\left[\begin{array}{cccccccc}
0 & \cdot & \cdot & 0 & 0 & \cdot & \cdot & \bullet \\
\cdot & \cdot & \cdot & \cdot & \cdot & \cdot & \cdot & \cdot \\
\cdot & \cdot & \cdot & \cdot & \cdot & \cdot & \cdot & \cdot \\
0 & \cdot & \cdot & 0 & \bullet & \cdot & \cdot & \bullet \\
0 & \cdot & \cdot & \bullet & \bullet & \cdot & \cdot & \bullet \\
\cdot & \cdot & \cdot & \cdot & \cdot & \cdot & \cdot & \cdot \\
\cdot & \cdot & \cdot & \cdot & \cdot & \cdot & \cdot & \cdot \\
\bullet & \cdot & \cdot & \bullet & \bullet & \cdot & \cdot & \bullet
\end{array}\right]}
\end{aligned}
$$

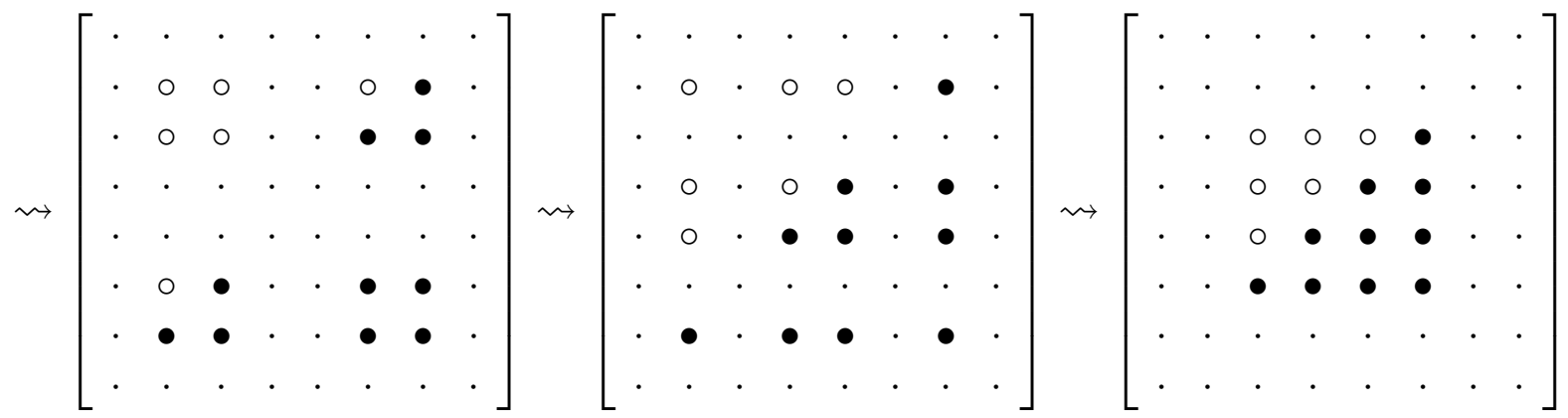

Unfortunately, as for the method by Bunse-Gerstner/Faßbender, there is no convergence proof for JIGH4, but convergence can be observed in experiments.

\section{A method working on 2-by-2 subproblems (JIGH2)}

In this section, we discuss the algorithm JIGH2 (Jacobi-like algorithm for the indefinite generalized Hermitian eigenvalue problem based on $\underline{2} \times 2$ subproblems). But first, let us have another look at the algorithm JIGH4. Why do we consider $4 \times 4$ subproblems instead of $2 \times 2$ subproblems? This comes from the fact that we want to deal with subproblems which have a structure corresponding to the one of the original problem. For example, if we consider the $8 \times 8$ pencil sketched below, then the $4 \times 4$ subpencil indicated by the bold discs is the smallest Hermitian subpencil that contains the $(2,3)$-entry ' $*$ ' of the pencil and that may be used to transport 'weight' from the upper anti-triangular part of the pencil into the lower anti-triangular part.

$$
\lambda \mathcal{G}-\mathcal{H}=\left[\begin{array}{cccccccc}
\cdot & \cdot & \cdot & \cdot & \cdot & \cdot & \cdot & \cdot \\
\cdot & \bullet & * & \cdot & \cdot & \bullet & \bullet & \cdot \\
\cdot & \bullet & \bullet & \cdot & \cdot & \bullet & \bullet & \cdot \\
\cdot & \cdot & \cdot & \cdot & \cdot & \cdot & \cdot & \cdot \\
\cdot & \cdot & \cdot & \cdot & \cdot & \cdot & \cdot & \cdot \\
\cdot & \bullet & \bullet & \cdot & \cdot & \bullet & \bullet & \cdot \\
\cdot & \bullet & \bullet & \cdot & \cdot & \bullet & \bullet & \cdot \\
\cdot & \cdot & \cdot & \cdot & \cdot & \cdot & \cdot & \cdot
\end{array}\right]
$$

Thus, if we want to work on $2 \times 2$ subproblems, some of them have to be non-Hermitian. 
Suppose that we want to eliminate the $(k, l)$-entry (and thus, simultaneously the $(l, k)$ entry) of the pencil in one Jacobi step, where we assume $k<l \leq n$. This entry is contained in the $2 \times 2$ subpencil

$$
\lambda \tilde{\mathcal{G}}-\tilde{\mathcal{H}}=\lambda\left[\begin{array}{cc}
g_{k l} & g_{k, 2 n+1-k} \\
g_{2 n+1-l, l} & g_{2 n+1-l, 2 n+1-k}
\end{array}\right]-\left[\begin{array}{cc}
h_{k l} & h_{k, 2 n+1-k} \\
h_{2 n+1-l, l} & h_{2 n+1-l, 2 n+1-k}
\end{array}\right]
$$

which we assume to be regular. This subpencil is indicated by bold discs in the sketch (9) below for the $8 \times 8$ case with $(k, l)=(2,3)$.

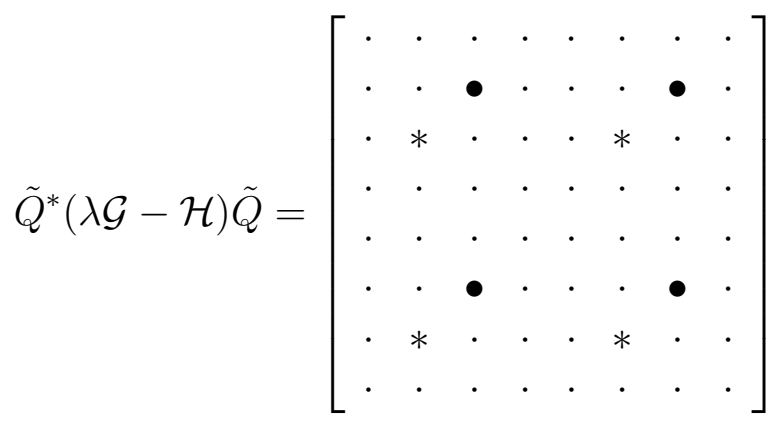

(Analogously, the subpencil indicated by stars contains the $(3,2)$-entry, but for the moment, we ignore this subpencil.) Clearly, the subpencil (8) is no longer Hermitian, but we can use it to transport 'weight' from the upper anti-triangular part of the pencil into the lower anti-triangular part by applying an anti-triangular version of the generalized Schur decomposition, i.e., we compute unitary matrices $U=\left(u_{i j}\right)$ and $V=\left(v_{i j}\right)$ such that $U(\lambda \tilde{\mathcal{G}}-\tilde{\mathcal{H}}) V$ is in anti-triangular form. Indeed, if $w=\left(w_{1}, w_{2}\right)^{T}$ is a normalized eigenvector of $\lambda \tilde{\mathcal{G}}-\tilde{\mathcal{H}}$ and if $x=\left(x_{1}, x_{2}\right)^{T}=\tilde{\mathcal{G}} w /\|\tilde{\mathcal{G}} w\|\left(\right.$ or $x=\left(x_{1}, x_{2}\right)^{T}=\tilde{\mathcal{H}} w /\|\tilde{\mathcal{H}} w\|$ if $\left.\tilde{\mathcal{G}} w=0\right)$, then

$$
U=\left[\begin{array}{cc}
-x_{2} & x_{1} \\
\bar{x}_{1} & \bar{x}_{2}
\end{array}\right], \quad V=\left[\begin{array}{cc}
w_{1} & -\bar{w}_{2} \\
w_{2} & \bar{w}_{1}
\end{array}\right]
$$

are unitary and $U(\lambda \tilde{\mathcal{G}}-\tilde{\mathcal{H}}) V$ is in anti-triangular form.

In order to preserve the structure of the Hermitian pencil, we define $\tilde{Q}$ to be the matrix that differs from the identity $I_{2 n}$ only in the submatrix

$$
\tilde{Q}_{k l}^{(4)}=Q=\left[\begin{array}{cccc}
\bar{u}_{11} & 0 & \bar{u}_{21} & 0 \\
0 & v_{11} & 0 & v_{12} \\
\bar{u}_{12} & 0 & \bar{u}_{22} & 0 \\
0 & v_{21} & 0 & v_{22}
\end{array}\right] \quad \text { or } \quad \tilde{Q}_{k l}^{(4)}=Q=\left[\begin{array}{cccc}
\bar{u}_{11} & \bar{u}_{21} & 0 & 0 \\
\bar{u}_{12} & \bar{u}_{22} & 0 & 0 \\
0 & 0 & v_{11} & v_{12} \\
0 & 0 & v_{21} & v_{22}
\end{array}\right]
$$

if $l<n+1-l$ or $l>n+1-l$, respectively. (Note that the case $l=n+1-l$ cannot occur if $n$ is even.) Then, we transform the pencil via $\tilde{Q}^{*}(\lambda \mathcal{G}-\mathcal{H}) \tilde{Q}$. To illustrate the effect of this simultaneous similarity transformation, we again refer to the sketch in (9). Note that in the updated pencil, the subpencil indicated by the bold discs in $(9)$ is just $U(\lambda \tilde{\mathcal{G}}-\tilde{\mathcal{H}}) V$. Since we used a structure preserving transformation, also the subpencil indicated by the stars is transformed to anti-triangular form. From this point of view, we again worked on 
a $4 \times 4$ subpencil, but the transformation was computed by solving an (unstructured) $2 \times 2$ problem only. We note that the unstructured subpencil $\lambda \tilde{\mathcal{G}}-\tilde{\mathcal{H}}$ can always be reduced to anti-triangular form provided that the subpencil is regular. This is different if we want to eliminate the $(k, k)$-entry of the Hermitian pencil, where $k \leq n$. Then, we have to consider the $2 \times 2$ Hermitian subpencil

$$
\lambda\left[\begin{array}{cc}
g_{k k} & g_{k, 2 n+1-k} \\
\bar{g}_{k, 2 n+1-k} & g_{2 n+1-k, 2 n+1-k}
\end{array}\right]-\left[\begin{array}{cc}
h_{k k} & h_{k, 2 n+1-k} \\
\bar{h}_{k, 2 n+1-k} & h_{2 n+1-k, 2 n+1-k}
\end{array}\right]
$$

and if this pencil has real eigenvalues, then an anti-triangular form under simultaneous unitary similarity need not exist. (In this case, we do not transform the subpencil at all.) For the rest of the paper, if $X=\left(x_{i j}\right) \in \mathbb{C}^{2 n \times 2 n}$, let $X_{k l}^{(2)}$ denote the matrix

$$
X_{k l}^{(2)}=\left[\begin{array}{cc}
x_{k l} & x_{k, 2 n+1-k} \\
x_{2 n+1-l, l} & x_{2 n+1-l, 2 n+1-k}
\end{array}\right] .
$$

During the computation of the $2 \times 2$ anti-triangular forms, there are two choices for the transformation matrices. As a strategy, we suggest to choose the transformation matrices that are closest to the identity, i.e., in the computations, we start with the (normalized) eigenvector that is closest to the first unit vector.

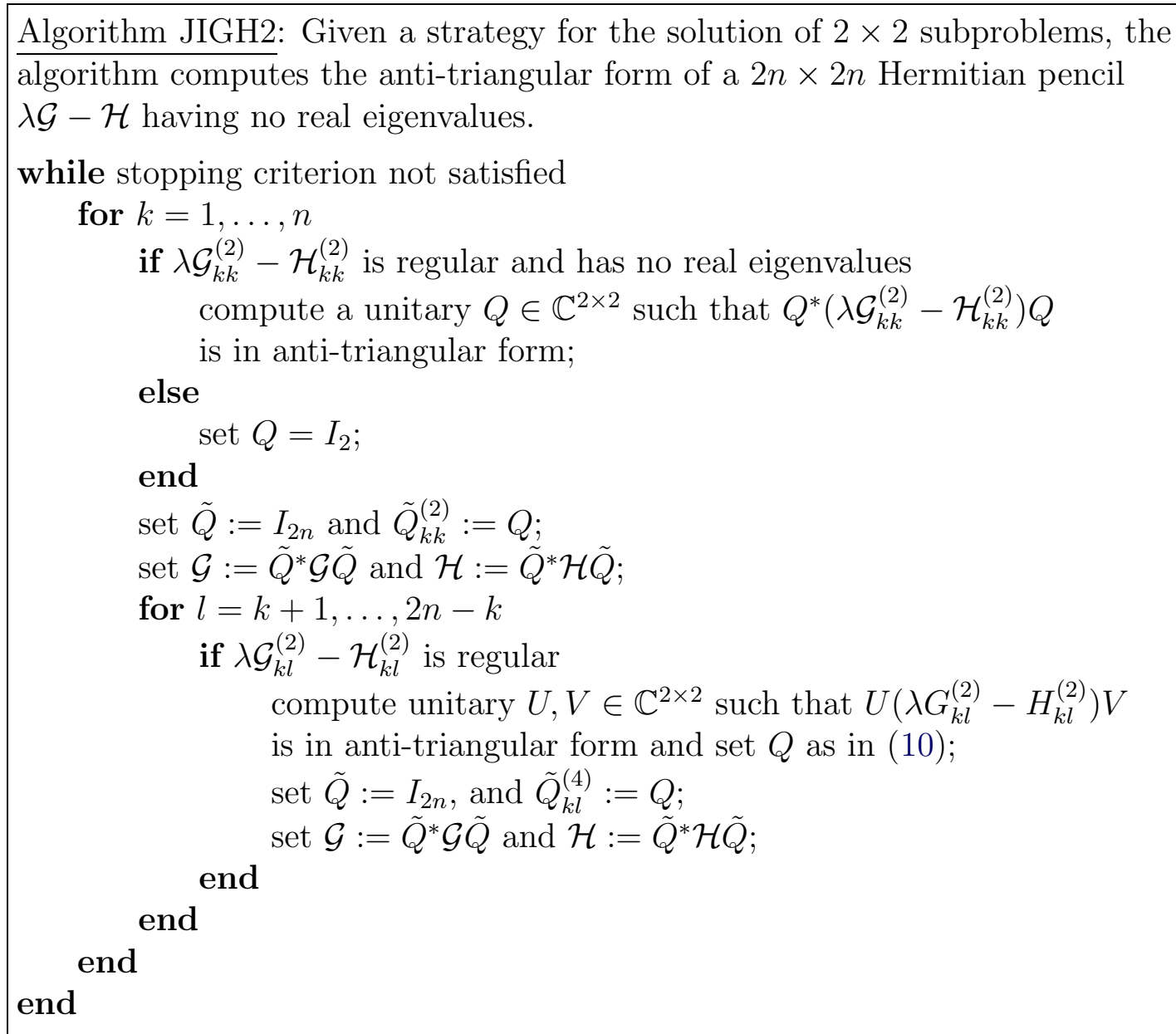


Again, a 'sweep' will denote one complete performance of the 'while'-loop. Assuming that all Hermitian $2 \times 2$ problems can be solved, a sweep in the $6 \times 6$ case proceeds as indicated in the sketch below. Again, the current subpencil is marked by the bold and blank discs, where the blank discs display the elements that will be annihilated in the current step. The subpencil marked by the stars will also be transformed to anti-triangular form for symmetry reasons.

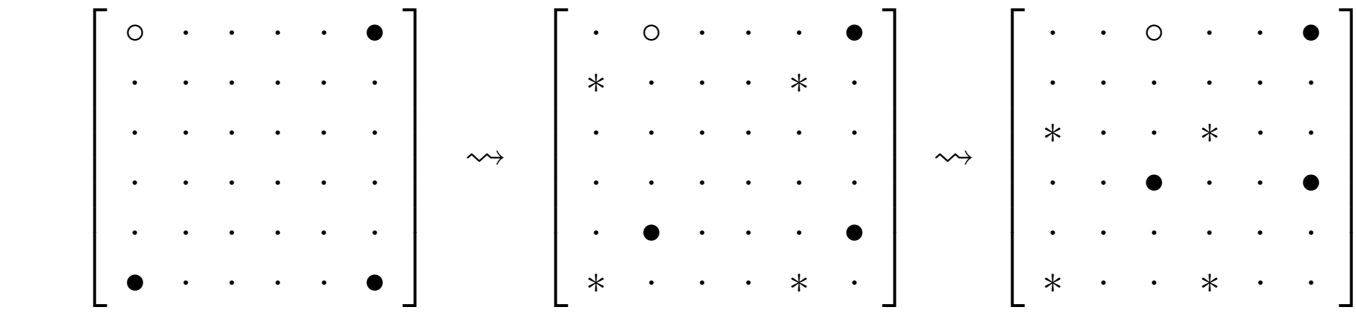

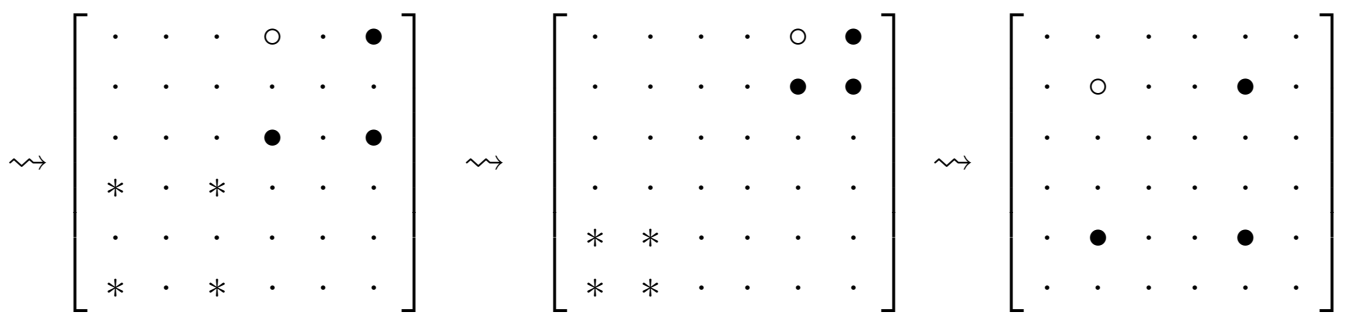

$$
\begin{aligned}
& \rightsquigarrow\left[\begin{array}{cccccc}
\cdot & \cdot & \cdot & \cdot & \cdot & \cdot \\
\cdot & \cdot & \circ & \cdot & \bullet & \cdot \\
\cdot & * & \cdot & * & \cdot & \cdot \\
\cdot & \cdot & \bullet & \cdot & \bullet & \cdot \\
\cdot & * & \cdot & * & \cdot & \cdot \\
\cdot & \cdot & \cdot & \cdot & \cdot & \cdot
\end{array}\right] \rightsquigarrow\left[\begin{array}{cccccc}
\cdot & \cdot & \cdot & \cdot & \cdot & \cdot \\
\cdot & \cdot & \cdot & 0 & \bullet & \cdot \\
\cdot & \cdot & \cdot & \bullet & \bullet & \cdot \\
\cdot & * & * & \cdot & \cdot & \cdot \\
\cdot & * & * & \cdot & \cdot & \cdot \\
\cdot & \cdot & \cdot & \cdot & \cdot & \cdot
\end{array}\right] \rightsquigarrow\left[\begin{array}{cccccc}
\cdot & \cdot & \cdot & \cdot & \cdot & \cdot \\
\cdot & \cdot & \cdot & \cdot & \cdot & \cdot \\
\cdot & \cdot & 0 & \bullet & \cdot & \cdot \\
\cdot & \cdot & \bullet & \bullet & \cdot & \cdot \\
\cdot & \cdot & \cdot & \cdot & \cdot & \cdot \\
\cdot & \cdot & \cdot & \cdot & \cdot & \cdot
\end{array}\right]
\end{aligned}
$$

Since JIGH2 works on $2 \times 2$ subproblems rather than on $4 \times 4$ problems, it can be considered a direct generalization of Eberlein's method [7].

\section{Proof of local convergence of JIGH2}

In this section we proof that JIGH2 converges quadratically, if the the given Hermitian pencil $\lambda \mathcal{G}-\mathcal{H}$ is already sufficiently close to being in anti-triangular form and if for the solution of $2 \times 2$ subproblems the strategy is used that chooses the transformation matrices that are closest to the identity. But first, let us introduce some notation which is adopted from [27] (see also [29]). Given two pairs $\left(a_{1}, b_{1}\right),\left(a_{2}, b_{2}\right) \in(\mathbb{C} \times \mathbb{C}) \backslash\{(0,0)\}$, let

$$
\operatorname{dif}\left(\left(a_{1}, b_{1}\right),\left(a_{2}, b_{2}\right)\right):=\inf _{p, q: \max (|p|,|q|)=1} \max \left(p a_{1}+q a_{2}, p b_{1}+q b_{2}\right) .
$$

Then by [29] Theorem VI.1.11 we have that $\operatorname{dif}\left(\left(a_{1}, b_{1}\right),\left(a_{2}, b_{2}\right)\right)>0$ if and only if the spectra of the $1 \times 1$ pencils $\lambda a_{1}-b_{1}$ and $\lambda a_{2}-b_{2}$ are disjoint. 
Lemma 3 Let $\varepsilon, \eta>0$ and

$$
\lambda \mathcal{G}-\mathcal{H}=\lambda\left[\begin{array}{ll}
g_{11} & g_{12} \\
\bar{g}_{12} & g_{22}
\end{array}\right]-\left[\begin{array}{ll}
h_{11} & h_{12} \\
\bar{h}_{12} & h_{22}
\end{array}\right]
$$

be such that $\left|g_{11}\right|,\left|h_{11}\right|<\varepsilon,\left|g_{22}\right|,\left|h_{22}\right|<\eta$, and

$$
\varrho=\operatorname{dif}\left(\left(g_{12}, h_{12}\right),\left(\bar{g}_{12}, \bar{h}_{12}\right)\right)>0 .
$$

If $4 \eta \varepsilon / \varrho^{2}<1$, then $\lambda \mathcal{G}-\mathcal{H}$ has no real eigenvalues and, in particular, $\lambda \mathcal{G}-\mathcal{H}$ can be transformed to anti-triangular form.

Proof. With the assumptions above, $\lambda \mathcal{G}-\mathcal{H}$ has two distinct eigenvalues by [29] Theorem VI.2.13. For $t \in \mathbb{R}$ consider the pencil

$$
P(t):=\lambda\left[\begin{array}{cc}
t g_{11} & g_{12} \\
\bar{g}_{12} & g_{22}
\end{array}\right]-\left[\begin{array}{cc}
t h_{11} & h_{12} \\
\bar{h}_{12} & h_{22}
\end{array}\right] .
$$

Then $P(t)$ is a regular Hermitian pencil for all $t \in[0,1]$ (this follows by investigating its determinant) and $P(1)=\lambda \mathcal{G}-\mathcal{H}$. Since $\varrho>0$, the pencil $P(0)$ has two complex conjugate eigenvalues. Assume that the eigenvalues of $P(1)$ are real. Consider

$$
\tilde{t}:=\inf _{t \in[0,1]}\{t: P(t) \text { has real eigenvalues }\} \text {. }
$$

Since the eigenvalues of regular pencils depend continously on the entries of the pencil, we obtain that $P(\tilde{t})$ has a real eigenvalue with multiplicity two. Since $\left|\tilde{t} g_{11}\right|,\left|\tilde{t} h_{11}\right|<\varepsilon$, this contradicts Theorem VI.2.13. in [29]. (This theorem states, in particular, that the eigenvalues must be distinct.) Thus, $\lambda \mathcal{G}-\mathcal{H}$ cannot have real eigenvalues.

Before we show local convergence of JIGH2, let us analyze what happens in one single Jacobi-step of the algorithm. Let us assume that we are in the $\mu$ th sweep and that we perform the $p$ th Jacobi step in this sweep. Since any sweep consists of

$$
s:=\frac{n^{2}}{4}=\sum_{k=1}^{n / 2}(2 k-1)
$$

Jacobi steps, we are performing step number $\nu:=\mu s+p$, currently working on the updated pencil $\lambda \mathcal{G}_{\nu}-\mathcal{H}_{\nu}, \mathcal{G}_{\nu}=\left(g_{i j}^{(\nu)}\right), \mathcal{H}_{\nu}=\left(h_{i j}^{(\nu)}\right)$, where $\lambda \mathcal{G}_{0}-\mathcal{H}_{0}:=\lambda \mathcal{G}-\mathcal{H}$. Assume, furthermore, that we want to eliminate the $(k, l)$-entry (and the $(l, k)$-entry if $k \neq l)$ of the current pencil $\lambda \mathcal{G}_{\nu}-\mathcal{H}_{\nu}$, where $k \leq l \leq n$. Let

$$
\begin{aligned}
\delta_{\nu} & :=\max \left\{\left|g_{i j}^{(\nu)}\right|,\left|h_{i j}^{(\nu)}\right| \mid i<k \text { or }(i=k \text { and } i \leq j<l)\right\}, \\
\varepsilon_{\nu} & :=\max \left\{\left|g_{i j}^{(\nu)}\right|,\left|h_{i j}^{(\nu)}\right| \mid i+j \leq n\right\}, \\
\eta_{\nu} & :=\max \left\{\left|g_{i j}^{(\nu)}\right|,\left|h_{i j}^{(\nu)}\right| \mid i+j \geq n+1\right\}, \\
\varrho_{\nu} & :=\min _{i \neq j} \operatorname{dif}\left(\left(g_{i, n+1-i}^{(\nu)}, h_{i, n+1-i}^{(\nu)}\right),\left(g_{j, n+1-j}^{(\nu)}, h_{j, n+1-j}^{(\nu)}\right)\right),
\end{aligned}
$$


where we assume $\varrho_{\nu}>0$ and $4 \varepsilon_{\nu} \eta_{\nu} / \varrho_{\nu}^{2}<1$. Thus, $\varepsilon_{\nu}$ and $\eta_{\nu}$, respectively, are the largest absolute values of entries of $\mathcal{G}_{\nu}$ and $\mathcal{H}_{\nu}$ in the strict upper anti-triangular part or in the lower anti-triangular part (including the anti-diagonal), respectively, while $\delta_{\nu}$ is the largest absolute value of entries in the strict upper anti-triangular part that have already been considered (and possibly annihilated) once in the current sweep. In the following, we will distinguish two cases.

Case (1) $k=l$. Then the current step of JIGH2 computes a unitary $Q \in \mathbb{C}^{2 \times 2}$ such that

$$
Q^{*}\left(\lambda\left[\begin{array}{cc}
g_{k k} & g_{k, n+1-k} \\
\bar{g}_{k, n+1-k} & g_{n+1-k, n+1-k}
\end{array}\right]-\left[\begin{array}{cc}
h_{k k} & h_{k, n+1-k} \\
\bar{h}_{k, n+1-k} & h_{n+1-k, n+1-k}
\end{array}\right]\right) Q
$$

is in anti-triangular form. Note that such a $Q$ exists by Lemma 3. Clearly, $Q$ can be taken of the form

$$
Q=\left[\begin{array}{cc}
\cos x & -e^{-i \alpha} \sin x \\
e^{i \alpha} \sin x & \cos x
\end{array}\right]
$$

for some $x, \alpha \in \mathbb{R}$. By Theorem VI.2.13 in [29], we then obtain $|\sin x|<2 \varepsilon_{\nu} / \varrho_{\nu}$. Note that only elements in the $k$ th and $(n+1-k)$ th rows and columns of the updated pencil $\lambda G_{\nu+1}-H_{\nu+1}$ have been changed. For $i<k$, we obtain

$$
\left|g_{i k}^{(\nu+1)}\right|=\left|g_{i k}^{(\nu)} \cos x+g_{i, n+1-k}^{(\nu)} e^{i \alpha} \sin x\right| \leq\left|g_{i k}^{(\nu)}\right|+\left|g_{i, n+1-k}^{(\nu)}\right| \cdot|\sin x|<\delta_{\nu}\left(1+2 \frac{\varepsilon_{\nu}}{\varrho_{\nu}}\right) .
$$

The same bound holds for $\left|h_{i k}^{(\nu+1)}\right|$. Similarly, we obtain for $k<i<n+1-k$ that

$$
\left|g_{i k}^{(\nu+1)}\right|,\left|h_{i k}^{(\nu+1)}\right|<\varepsilon_{\nu}\left(1+2 \frac{\eta_{\nu}}{\varrho_{\nu}}\right) .
$$

Moreover, we obtain

$$
\begin{aligned}
& g_{n+1-k, k}^{(\nu+1)} \\
= & -g_{k k}^{(\nu)} e^{i \alpha} \sin x \cos x+g_{n+1-k, k}^{(\nu)} \cos ^{2} x-g_{k, n+1-k}^{(\nu)}\left(e^{i \alpha} \sin x\right)^{2}+g_{n+1-k, n+1-k}^{(\nu)} e^{i \alpha} \sin x \cos x \\
= & g_{n+1-k, k}^{(\nu)}+\Delta_{k}, \quad\left(\text { using } \cos ^{2} x=1-\sin ^{2} x\right)
\end{aligned}
$$

where

$$
\left|\Delta_{k}\right|<\varepsilon_{\nu} \cdot 2 \frac{\varepsilon_{\nu}}{\varrho_{\nu}}+\eta_{\nu} \cdot 4 \frac{\varepsilon_{\nu}^{2}}{\varrho_{\nu}^{2}}+\eta_{\nu} \cdot 4 \frac{\varepsilon_{\nu}^{2}}{\varrho_{\nu}^{2}}+\eta_{\nu} \cdot 2 \frac{\varepsilon_{\nu}}{\varrho_{\nu}}=2\left(\frac{\varepsilon_{\nu}^{2}}{\varrho_{\nu}}+4 \eta_{\nu} \frac{\varepsilon_{\nu}^{2}}{\varrho_{\nu}^{2}}+\eta_{\nu} \frac{\varepsilon_{\nu}}{\varrho_{\nu}}\right) .
$$

A corresponding result holds for $h_{n+1-k, k}^{(\nu+1)}$. Then the inequality

$\operatorname{dif}\left(\left(a_{1}+e_{1}, b_{1}+f_{1}\right),\left(a_{2}+e_{2}, b_{2}+f_{2}\right)\right) \geq \operatorname{dif}\left(\left(a_{1}, b_{1}\right),\left(a_{2}, b_{2}\right)\right)-\max \left(\left|e_{1}\right|+\left|e_{2}\right|,\left|f_{1}\right|+\left|f_{2}\right|\right)$,

see formula (2.19) in [29], implies

$$
\operatorname{dif}\left(\left(g_{n+1-i, i}^{(\nu+1)}, h_{n+1-i, i}^{(\nu+1)}\right),\left(g_{n+1-j, j}^{(\nu+1)}, h_{n+1-j, j}^{(\nu+1)}\right)\right)>\varrho_{\nu}-4\left(\frac{\varepsilon_{\nu}^{2}}{\varrho_{\nu}}+4 \eta_{\nu} \frac{\varepsilon_{\nu}^{2}}{\varrho_{\nu}^{2}}+\eta_{\nu} \frac{\varepsilon_{\nu}}{\varrho_{\nu}}\right)
$$


for all $i, j=1, \ldots, n ; i \neq j$.

Case (2) $k \neq l$. Then the current step of JIGH2 computes unitary matrices $P, Q \in \mathbb{C}^{2 \times 2}$ such that

$$
P^{*}\left(\lambda\left[\begin{array}{cc}
g_{k l}^{(\nu)} & g_{k, n+1-k}^{(\nu)} \\
g_{n+1-l, l}^{(\nu)} & g_{n+1-l, n+1-k}^{(\nu)}
\end{array}\right]-\left[\begin{array}{cc}
h_{k l}^{(\nu)} & h_{k, n+1-k}^{(\nu)} \\
h_{n+1-l, l}^{(\nu)} & h_{n+1-l, n+1-k}^{(\nu)}
\end{array}\right]\right) Q
$$

is in anti-triangular form. Without loss of generality, we may assume that $P$ and $Q$ have the forms

$$
P^{*}=\left[\begin{array}{cc}
\cos x & -e^{-i \alpha} \sin x \\
e^{i \alpha} \sin x & \cos x
\end{array}\right] \text { and } Q=\left[\begin{array}{cc}
\cos y & -e^{-i \beta} \sin y \\
e^{i \beta} \sin y & \cos y
\end{array}\right],
$$

where $x, y, \alpha, \beta \in \mathbb{R}$. By Theorem VI.2.13 in [29], we have $|\sin x|,|\sin y|<2 \varepsilon_{\nu} / \varrho_{\nu}$. Only elements of the $k$ th, $l$ th, $(n+1-l)$ th, and $(n+1-k)$ th rows and columns of $G_{\nu+1}$ and $H_{\nu+1}$ have been changed. If $l<n+1-l$, then we obtain that

$$
\begin{aligned}
& \left|g_{k k}^{(\nu+1)}\right| \\
= & \left|g_{k k}^{(\nu+1)} \cos ^{2} x-g_{n+1-l, k}^{(\nu+1)} e^{-i \alpha} \cos x \sin x-g_{k, n+1-l}^{(\nu+1)} e^{i \alpha} \cos x \sin x+g_{n+1-l, n+1-l}^{(\nu+1)} \sin ^{2} x\right| \\
< & \delta_{\nu}+\varepsilon_{\nu} \cdot 2 \frac{\varepsilon_{\nu}}{\varrho_{\nu}}+\varepsilon_{\nu} \cdot 2 \frac{\varepsilon_{\nu}}{\varrho_{\nu}}+\eta_{\nu} \cdot 4 \frac{\varepsilon_{\nu}^{2}}{\varrho_{\nu}^{2}}=\delta_{\nu}+4 \frac{\varepsilon_{\nu}^{2}}{\varrho_{\nu}}+4 \frac{\varepsilon_{\nu}^{2} \eta_{\nu}}{\varrho_{\nu}^{2}}
\end{aligned}
$$

and if furthermore $k<i<l$, then we obtain that

$$
\left|g_{i k}^{(\nu+1)}\right|=\left|g_{i k}^{(\nu)} \cos y-g_{i, n+1-l}^{(\nu)} e^{i \beta} \sin y\right|<\delta_{\nu}+2 \frac{\varepsilon_{\nu}^{2}}{\varrho_{\nu}} .
$$

By this and similar computations, we finally obtain for $i<k$ and $j \leq n+1-k$ that

$$
\left|g_{i j}^{(\nu+1)}\right|,\left|h_{i j}^{(\nu+1)}\right|<\delta_{\nu}\left(1+2 \frac{\varepsilon_{\nu}}{\varrho_{\nu}}\right) .
$$

For $i \geq k$ and for the case $l<n+1-l$ we obtain that $\left|g_{i j}^{(\nu+1)}\right|$ and $\left|h_{i j}^{(\nu+1)}\right|$ have the following upper bounds:

\begin{tabular}{c|c|c|c|} 
& $j=k$ & $j=l$ & $j=n+1-l$ \\
\hline$i=k$ & $\delta_{\nu}+4 \frac{\varepsilon_{\nu}^{2}}{\varrho_{\nu}}+4 \frac{\varepsilon_{\nu}^{2} \eta_{\nu}}{\varrho_{\nu}^{\nu}}$ & 0 & $\varepsilon_{\nu}\left(1+2 \frac{\delta_{\nu}+\eta_{\nu}}{\varrho_{\nu}}+4 \frac{\varepsilon_{\nu}^{2}}{\varrho_{\nu}^{2}}\right)$ \\
\hline$k<i<l$ & $\delta_{\nu}+2 \frac{\varepsilon_{\nu}^{2}}{\varrho_{\nu}}$ & $\varepsilon_{\nu}\left(1+2 \frac{\eta_{\nu}}{\varrho_{\nu}}\right)$ & $\varepsilon_{\nu}\left(1+2 \frac{\delta_{\nu}}{\varrho_{\nu}}\right)$ \\
\hline$i=l$ & 0 & $\varepsilon_{\nu}\left(1+4 \frac{\eta_{\nu}}{\varrho_{\nu}}+4 \frac{\varepsilon_{\nu} \eta_{\nu}}{\varrho_{\nu}}\right)$ & -- \\
\hline$l<i<n+1-l$ & $\varepsilon_{\nu}\left(1+2 \frac{\eta_{\nu}}{\varrho_{\nu}}\right)$ & $\varepsilon_{\nu}\left(1+2 \frac{\eta_{\nu}}{\varrho_{\nu}}\right)$ & -- \\
\hline$i=n+1-l$ & $\varepsilon_{\nu}\left(1+2 \frac{\delta_{\nu}+\eta_{\nu}}{\varrho_{\nu}}+4 \frac{\varepsilon_{\nu}^{2}}{\varrho_{\nu}^{2}}\right)$ & -- & -- \\
\hline$n+1-l$ & $\varepsilon_{\nu}\left(1+2 \frac{\eta_{\nu}}{\varrho_{\nu}}\right)$ & -- & -- \\
\hline$<i<n+1-k$ & \multicolumn{2}{c}{} \\
\hline
\end{tabular}


Analogously, we obtain for the case $l>n+1-l$ that $\left|g_{i j}^{(\nu+1)}\right|$ and $\left|h_{i j}^{(\nu+1)}\right|$ have the following upper bounds:

\begin{tabular}{c|c|c|c|} 
& $j=k$ & $j=n+1-l$ & $j=l$ \\
\hline$i=k$ & $\delta_{\nu}+4 \frac{\varepsilon_{\nu} \delta_{\nu}}{\varrho_{\nu}}+4 \frac{\varepsilon_{\nu}^{3}}{\varrho_{\nu}^{2}}$ & $\delta_{\nu}+2 \frac{\varepsilon_{\nu}^{2}+\varepsilon_{\nu} \delta_{\nu}}{\varrho_{\nu}}+4 \frac{\varepsilon_{\nu}^{2} \delta_{\nu}}{\varrho_{\nu}^{2}}$ & 0 \\
\hline$k<i<n+1-l$ & $\delta_{\nu}+2 \frac{\varepsilon_{\nu}^{2}}{\varrho_{\nu}}$ & $\varepsilon_{\nu}\left(1+2 \frac{\delta_{\nu}}{\varrho_{\nu}}\right)$ & $\varepsilon_{\nu}\left(1+2 \frac{\eta_{\nu}}{\varrho_{\nu}}\right)$ \\
\hline$i=n+1-l$ & $\delta_{\nu}+2 \frac{\varepsilon_{\nu}^{2}+\varepsilon_{\nu} \delta_{\nu}}{\varrho_{\nu}}+4 \frac{\varepsilon_{\nu}^{2} \delta_{\nu}}{\varrho_{\nu}^{2}}$ & $\varepsilon_{\nu}\left(1+4 \frac{\delta_{\nu}}{\varrho_{\nu}}+4 \frac{\varepsilon_{\nu} \delta_{\nu}}{\varrho_{\nu}^{2}}\right)$ & -- \\
\hline$n+1-l<i<l$ & $\delta_{\nu}+2 \frac{\varepsilon_{\nu}^{2}}{\varrho_{\nu}}$ & $\varepsilon_{\nu}\left(1+2 \frac{\delta_{\nu}}{\varrho_{\nu}}\right)$ & -- \\
\hline$i=l$ & 0 & -- & -- \\
\hline$l<i<n+1-k$ & $\varepsilon_{\nu}\left(1+2 \frac{\eta_{\nu}}{\varrho_{\nu}}\right)$ & -- & -- \\
\hline
\end{tabular}

Furthermore, we obtain that

$$
\begin{aligned}
g_{n+1-k, k}^{(\nu+1)} & =g_{n+1-k, k}^{(\nu)}+\Delta_{1}, \\
g_{n+1-l, l}^{(\nu+1)} & =g_{n+1-l, l}^{(\nu)}+\Delta_{2}, \\
h_{n+1-k, k}^{(\nu+1)} & =h_{n+1-k, k}^{(\nu)}+\Delta_{3}, \\
h_{n+1-l, l}^{(\nu+1)} & =h_{n+1-l, l}^{(\nu)}+\Delta_{4},
\end{aligned}
$$

where

$$
\left|\Delta_{1}\right|,\left|\Delta_{2}\right|,\left|\Delta_{3}\right|,\left|\Delta_{4}\right|<2\left(\frac{\varepsilon_{\nu}^{2}}{\varrho_{\nu}}+4 \eta_{\nu} \frac{\varepsilon_{\nu}^{2}}{\varrho_{\nu}^{2}}+\eta_{\nu} \frac{\varepsilon_{\nu}}{\varrho_{\nu}}\right) .
$$

From the analysis of both Case (1) and (2) and using $\delta_{\nu} \leq \varepsilon_{\nu} \leq \eta_{\nu}$ and $4 \varepsilon_{\nu} \eta_{\nu} / \varrho_{\nu}^{2}<1$, we find that

$$
\begin{array}{ll}
\delta_{\nu+1}=0, & \text { if } \nu \text { is a multiple of } \frac{n^{2}}{4} \\
\delta_{\nu+1}<\delta_{\nu}+4 \frac{\varepsilon_{\nu}^{2}}{\varrho_{\nu}}+4 \frac{\varepsilon_{\nu}^{2} \eta_{\nu}}{\varrho_{\nu}^{2}}, & \text { if } \nu \text { is not a multiple of } \frac{n^{2}}{4} \\
\varepsilon_{\nu+1}<\varepsilon_{\nu}\left(1+4 \frac{\eta_{\nu}}{\varrho_{\nu}}+4 \frac{\varepsilon_{\nu} \eta_{\nu}}{\varrho_{\nu}^{2}}\right)<\varepsilon_{\nu}\left(2+4 \frac{\eta_{\nu}}{\varrho_{\nu}}\right) & \\
\varrho_{\nu+1}>\varrho_{\nu}-4\left(\frac{\varepsilon_{\nu}^{2}}{\varrho_{\nu}}+4 \eta_{\nu} \frac{\varepsilon_{\nu}^{2}}{\varrho_{\nu}^{2}}+\eta_{\nu} \frac{\varepsilon_{\nu}}{\varrho_{\nu}}\right) .
\end{array}
$$

An analysis of the elements in the lower anti-triangular parts of $\mathcal{G}_{\nu}$ and $\mathcal{H}_{\nu}$ would also produce a bound for $\eta_{\nu+1}$, but for our purpose it is sufficient to note that $\eta_{\nu+1}$ is bounded by $\eta=\max \left(\|\mathcal{G}\|_{F},\|\mathcal{H}\|_{F}\right)$. Using the above, we will now show that JIGH2 is locally convergent and that the asymptotic convergence rate is quadratic. 
Theorem 4 Let $\lambda \mathcal{G}-\mathcal{H} \in \mathbb{C}^{n \times n}$ be a Hermitian pencil and for $\nu \in \mathbb{N} \cup\{0\}$, let $\delta_{\nu}, \varepsilon_{\nu}, \eta_{\nu}$, and $\varrho_{\nu}$ be defined as in (11)-(14). Moreover, let

$$
\eta:=\max \left(\|\mathcal{G}\|_{F},\|\mathcal{H}\|_{F}\right), \quad \varrho:=\frac{\varrho_{0}}{2}, \quad \text { and } \quad \varepsilon:=\varepsilon_{0}\left(2+4 \frac{\eta}{\varrho}\right)^{s}
$$

where $s:=\frac{n^{2}}{4}$. If $\varrho_{0}>0$ and if $\varepsilon_{0}$ is such small that

$$
\frac{\varepsilon \eta}{\varrho^{2}}<\frac{1}{4}, \quad \frac{\varepsilon^{2}}{\varrho}+4 \eta \frac{\varepsilon^{2}}{\varrho^{2}}+\eta \frac{\varepsilon}{\varrho} \leq \frac{1}{4 n^{2}}, \quad \text { and } \quad 2 n^{2} \varepsilon^{2}\left(\frac{1}{\varrho}+\frac{\eta}{\varrho^{2}}\right) \leq \varepsilon_{0},
$$

then there exists a constant $C>0$ such that $\varepsilon_{(\mu+1) s}<C \varepsilon_{\mu s}^{2}$ for all $\mu \in \mathbb{N} \cup\{0\}$.

Proof. From (17) and (18) we obtain that $\varepsilon_{\nu}$ (and $\varrho_{\nu}$, respectively) may increase (or decrease, respectively) in each Jacobi step. We first show by induction that this increase (decrease, respectively) remains under control, i.e., that for $\mu \in \mathbb{N} \cup\{0\}$ and $p=0, \ldots, s$, we have that

$$
\begin{aligned}
\varepsilon_{\mu s} \leq \frac{\varepsilon_{0}}{2^{\mu}}, & \varrho_{\mu s} \geq \varrho_{0}-\sum_{j=1}^{\mu} \frac{1}{2^{\mu+1}} \varrho_{0}>\varrho, \quad \text { and } \\
\varepsilon_{\mu s+p} \leq \varepsilon_{\mu s}\left(2+4 \frac{\eta}{\varrho}\right)^{p}, & \varrho_{\mu s+p} \geq \varrho_{\mu s}-p \frac{\varrho_{0}}{2^{\mu} n^{2}} .
\end{aligned}
$$

' $(\mu, p)=(0,0)$ ': There is nothing to prove.

' $(\mu, p) \Rightarrow(\mu, p+1)$ ': Let $p<s$. By the induction hypothesis for $(\mu, p)$ and $(\mu, 0)$, we have that

$$
\begin{aligned}
\varepsilon_{\mu s+p} & \leq \varepsilon_{\mu s}\left(2+4 \frac{\eta}{\varrho}\right)^{p} \leq \frac{\varepsilon_{0}}{2^{\mu}}\left(2+4 \frac{\eta}{\varrho}\right)^{p} \leq \frac{\varepsilon}{2^{\mu}} \\
\text { and } \quad \varrho_{\mu s+p} & \geq \varrho_{\mu s}-p \frac{\varrho_{0}}{2^{\mu} n^{2}} \geq \varrho_{0}-\sum_{j=1}^{\mu} \frac{1}{2^{j+1}} \varrho_{0}-\frac{n^{2}}{4} \frac{\varrho_{0}}{2^{\mu} n^{2}}=\varrho_{0}-\sum_{j=1}^{\mu+1} \frac{1}{2^{j+1}} \varrho_{0}>\varrho .
\end{aligned}
$$

Then we obtain from (17) and (18) that

$$
\begin{aligned}
\varepsilon_{\mu s+p+1} & <\varepsilon_{\mu s+p}\left(2+4 \frac{\eta_{\mu s+p}}{\varrho_{\mu s+p}}\right) \leq \varepsilon_{\mu s+p}\left(2+4 \frac{\eta}{\varrho}\right) \leq \varepsilon_{\mu s}\left(2+4 \frac{\eta}{\varrho}\right)^{p+1} \\
\varrho_{\mu s+p+1} & >\varrho_{\mu s+p}-4\left(\frac{\varepsilon_{\mu s+p}^{2}}{\varrho_{\mu s+p}}+4 \eta \frac{\varepsilon_{\mu s+p}^{2}}{\varrho_{\mu s+p}^{2}}+\eta \frac{\varepsilon_{\mu s+p}}{\varrho_{\mu s+p}}\right) \\
& \geq \varrho_{\mu s}-p \frac{\varrho_{0}}{2^{\mu} n^{2}}-4\left(\frac{\varepsilon^{2}}{\left(2^{\mu}\right)^{2} \varrho}+4 \eta \frac{\varepsilon^{2}}{\left(2^{\mu}\right)^{2} \varrho^{2}}+\eta \frac{\varepsilon}{2^{\mu} \varrho}\right) \\
& \geq \varrho_{\mu s}-p \frac{\varrho_{0}}{2^{\mu} n^{2}}-\frac{4}{2^{\mu}}\left(\frac{\varepsilon^{2}}{\varrho}+4 \eta \frac{\varepsilon^{2}}{\varrho^{2}}+\eta \frac{\varepsilon}{\varrho}\right) \\
& \geq \varrho_{\mu s}-(p+1) \frac{\varrho_{0}}{2^{\mu} n^{2}} . \quad(\text { by }(20))
\end{aligned}
$$


' $(\mu, p) \Rightarrow(\mu+1,0)$ ': For obtaining a bound for $\varepsilon_{(\mu+1) s}$, let us note that during the $(\mu+1)$ st sweep each entry in the strict upper anti-triangular part of the current pencil is set to zero at one step, and may then increase according to (16) during the rest of the sweep. Since $\varepsilon_{\mu s+p} \leq \varepsilon / 2^{\mu}$ and $\varrho_{\mu s+p} \geq \varrho$ for $p=0, \ldots, s$, we obtain by using (16) and (20) that

$$
\varepsilon_{(\mu+1) s}<\frac{n^{2}}{4}\left(\frac{\varepsilon}{2^{\mu}}\right)^{2}\left(\frac{4}{\varrho}+4 \frac{\eta}{\varrho^{2}}\right) \leq \frac{1}{2} \frac{\varepsilon_{0}}{\left(2^{\mu}\right)^{2}} \leq \frac{\varepsilon_{0}}{2^{\mu+1}} .
$$

This concludes the proof of (21) and (22). In particular, (22) implies that

$$
\varepsilon_{\mu s+p} \leq \varepsilon_{\mu s}\left(2+4 \frac{\eta}{\varrho}\right)^{s}
$$

Using this inequality instead of $\varepsilon_{\mu s+p} \leq \varepsilon / 2^{\mu}$, we obtain analogously to (23) that

$$
\varepsilon_{(\mu+1) s}<n^{2} \varepsilon_{\mu s}^{2}(2+4 \eta / \varrho)^{2 s}\left(\frac{1}{\varrho}+\frac{\eta}{\varrho^{2}}\right)=C \varepsilon_{\mu s}^{2},
$$

where $C$ only depends on $n, \eta$, and $\varrho$.

We note that the main purpose of the proof of Theorem 4 is to show asymptotic quadratic convergence and not to produce optimal estimation bounds. ence, for the sake of simplification of the discussion, we did some rather rough estimates. A more detailed investigation could certainly produce better bounds, but this would complicate our discussion considerably.

\section{A modified method (MJIGH2)}

Unfortunately, JIGH2 does not converge globally. Consider the following example:

$$
\lambda \mathcal{G}-\mathcal{H}=\lambda\left[\begin{array}{llll}
0 & 0 & 0 & 1 \\
0 & 0 & 1 & 0 \\
0 & 1 & 0 & 0 \\
1 & 0 & 0 & 0
\end{array}\right]-\left[\begin{array}{cccc}
2 & 0 & 0 & i \\
0 & 0 & 2 i & 1 \\
0 & -2 i & -4 & 0 \\
-i & 1 & 0 & 1
\end{array}\right]
$$

Then this pencil has the spectrum $\left\{ \pm \frac{1}{2}+\sqrt{\frac{7}{4}} i, \pm \frac{1}{2}-\sqrt{\frac{7}{4}} i\right\}$, whereas the subpencil

$$
\lambda\left[\begin{array}{ll}
0 & 1 \\
1 & 0
\end{array}\right]-\left[\begin{array}{cc}
2 & i \\
-i & 1
\end{array}\right]
$$

has the spectrum $\{1,-1\}$. Thus, JIGH2 stagnates although the pencil can be reduced to anti-triangular form. The phenomenom of stagnation also occurs during numerical experiments. This problem can be solved by slightly modifying the algorithm JIGH2. Note that if during one sweep of JIGH2, we encounter a $2 \times 2$-subpencil with real eigenvalues (and thus, there is a chance of stagnation of JIGH2), then this $2 \times 2$-subpencil may be 
contained in a $4 \times 4$-subpencil that has (at least two) nonreal eigenvalues. In this case, performing Jacobi-steps as in JIGH4 may avoid stagnation of the method. This idea motivates the following modification of JIGH2 presented below that we will call MJIGH2

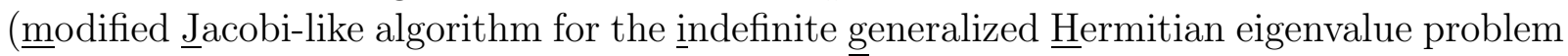
based on $\underline{2} \times 2$ subproblems).

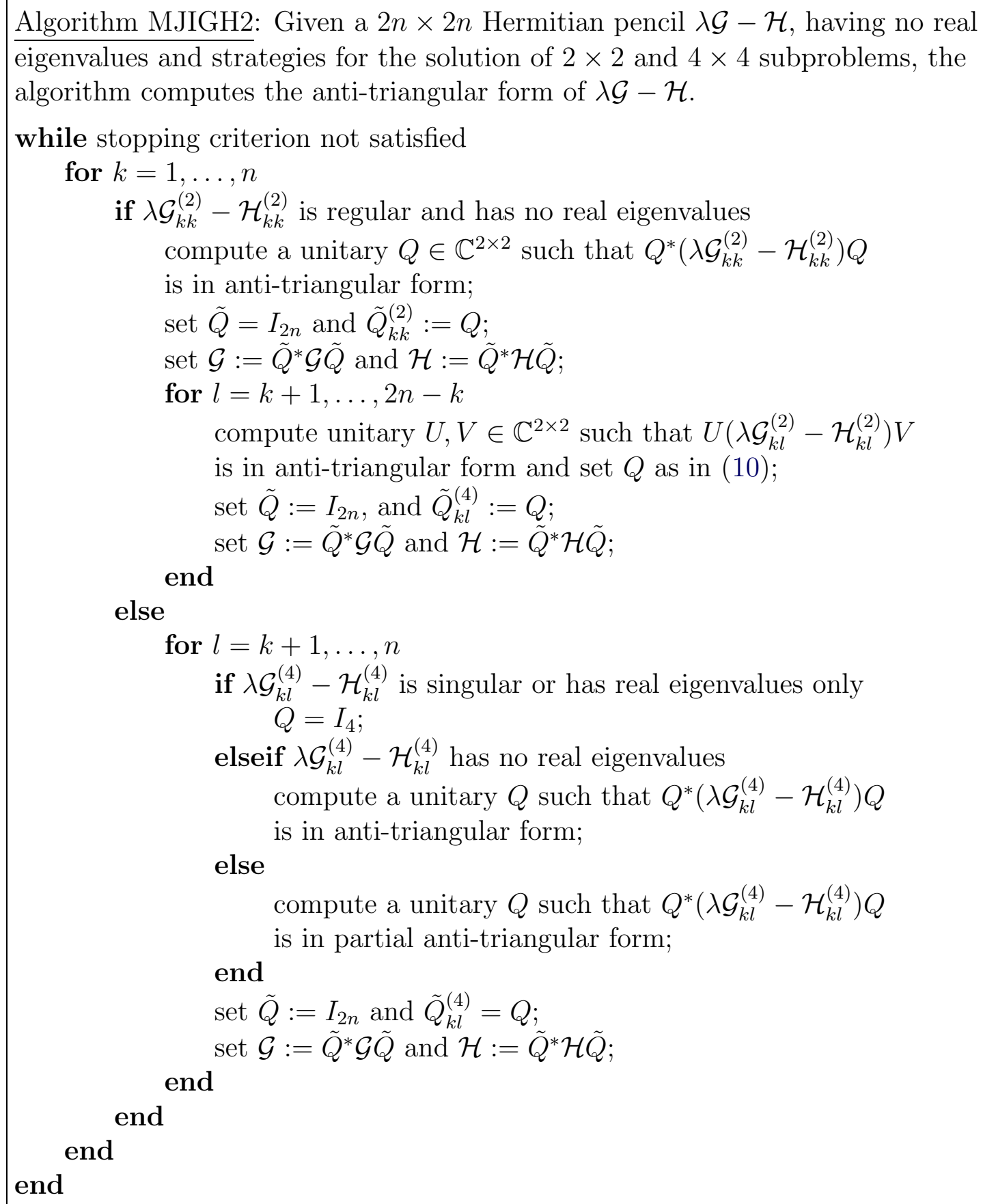


Thus, whenever a Hermitian $2 \times 2$ subproblems having real eigenvalues occurs, we continue the second 'for'-loop by solving $4 \times 4$ subproblems instead of $2 \times 2$ problems. As seen in Section 5, no $2 \times 2$ Hermitian subproblems having only real eigenvalues occur if $\lambda \mathcal{G}-\mathcal{H}$ is sufficiently close to being in anti-triangular form and thus, MJIGH2 reduces to JIGH2 in this case. This means that MJIGH2 has the same local convergence properties as JIGH2. In addition, convergence has been observed for all test examples in the current research.

\section{Numerical experiments}

For numerical tests, the algorithms JIGH4 and MJIGH2 were implemented in MATLAB Version 5.3 and run on a PC with a Pentium III processor $(800 \mathrm{MHz})$. The relative machine precision was eps $=2.2204 \times 10^{-16}$. The strategies used for the solution of the $4 \times 4$ and $2 \times 2$ subproblems were the ones explained in Section 3.2 and Section 4. Given a Hermitian pencil $\lambda \mathcal{G}-\mathcal{H} \in \mathbb{C}^{n \times n}, \mathcal{G}=\left(g_{i j}\right), \mathcal{H}=\left(h_{i j}\right)$, with

$$
\operatorname{norm}(\mathcal{G}, \mathcal{H}):=\sqrt{\|\mathcal{G}\|_{F}^{2}+\|\mathcal{H}\|_{F}^{2}}=1
$$

we chose as a stopping criterion

$$
e(\mathcal{G}, \mathcal{H}):=\max \left\{\left|g_{i j}\right|,\left|h_{i j}\right| \mid i+j \leq n\right\}<50 \text { eps }
$$

(In practice, one should compare $\left|g_{i j}\right|,\left|h_{i j}\right|$ with $\left|g_{n+1-i, i}\right|,\left|h_{n+1-i, i}\right|,\left|g_{n+1-j, j}\right|,\left|h_{n+1-j, j}\right|$, $\left|g_{n+1-i, n+1-j}\right|$, and $\left|h_{n+1-i, n+1-j}\right|$ generalizing the componentwise error analysis for Jacobi's algorithm for positive definite matrices in [20]. This topic is currently under investigation.)

A first test was run for 50 randomly generated $2 n \times 2 n$ Hermitian pencils $\lambda \mathcal{G}-\mathcal{H}$ having no real eigenvalues and with $\operatorname{norm}(\mathcal{G}, \mathcal{H})=1$. This test was run over different dimensions $n=3,4, \ldots, 9,10,15,20,25,30$. Convergence of both JIGH4 and MJIGH2 has been observed for all test problems. Figure 1 shows the average number of sweeps necessary for convergence. Note that except for the case $n=3$, this number was always lower with MJIGH2 than with JIGH4.

Figure 2 shows the typical convergence behavior of both methods for the case $n=10$, i.e., for a randomly generated $20 \times 20$ Hermitian pencil. At the beginning both methods show a similar behavior. In both cases, the convergence rate is not necessarily monotone, but, as soon as the pencil tends to being close to anti-triangular form, the convergence rate of MJIGH2 becomes quadratic very fast, while the one of JIGH4 is somewhere in between linear and quadratic, slowly approaching quadratic convergence towards the end of the process.

Thus, MJIGH2 converges faster than JIGH4. But, for a fair comparison, we also have to compare the cost per sweep of each method. Since an estimate of flop count is difficult to obtain (it depends on the nature of the eigenvalues of the $4 \times 4$ and $2 \times 2$ subpencils), the average flop count per sweep has been determined experimentally and is presented in the left graph of Figure 3 for both JIGH2 and JIGH4. 


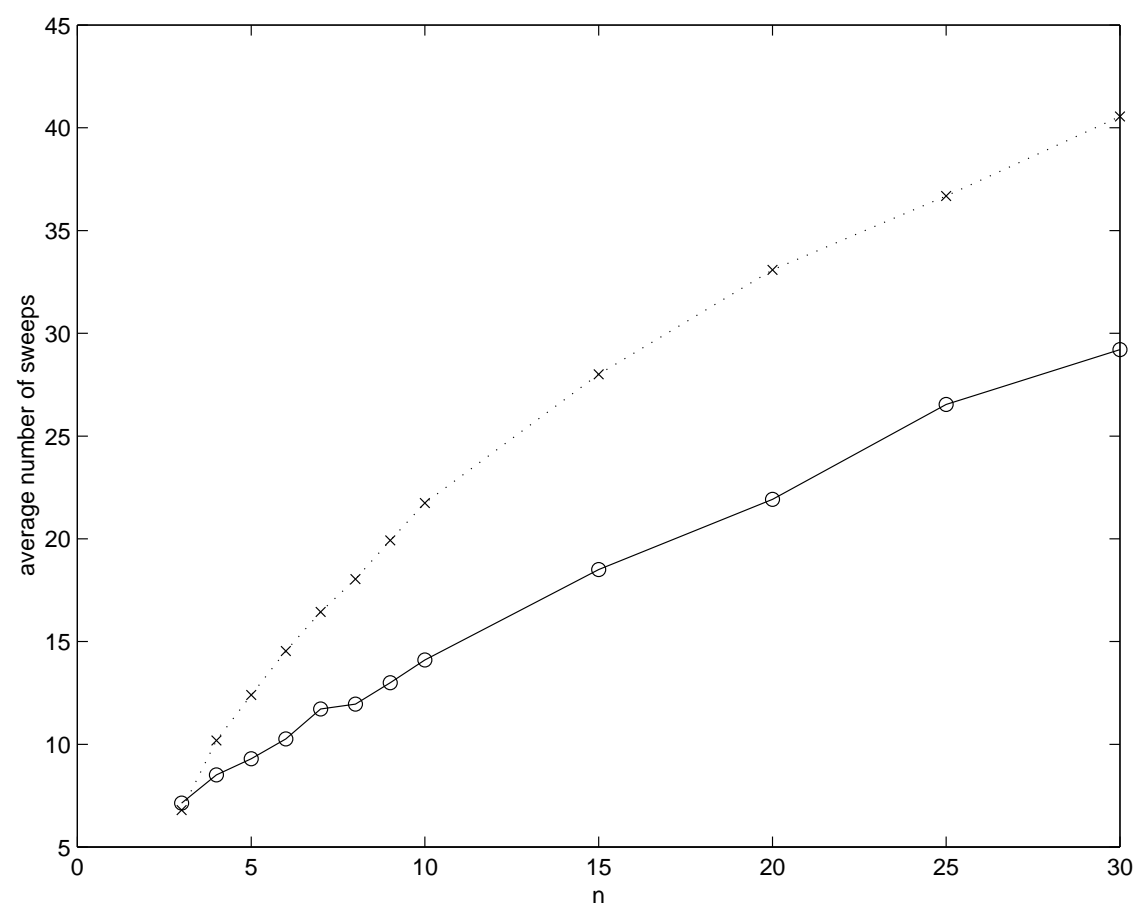

Figure 1: 50 randomly generated Hermitian pencils, '—' MJIGH2, '. .' JIGH4

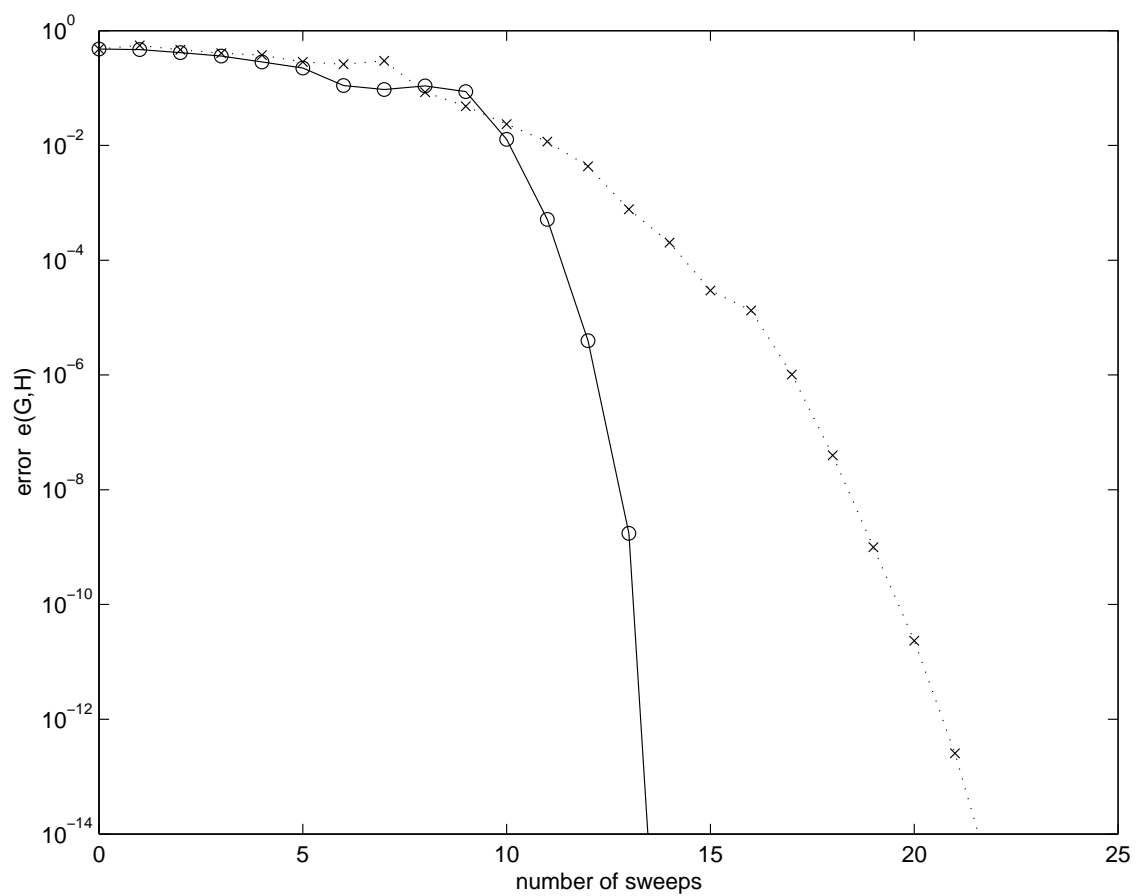

Figure 2: Typical convergence behavior, '—' MJIGH2, '. .' JIGH4 

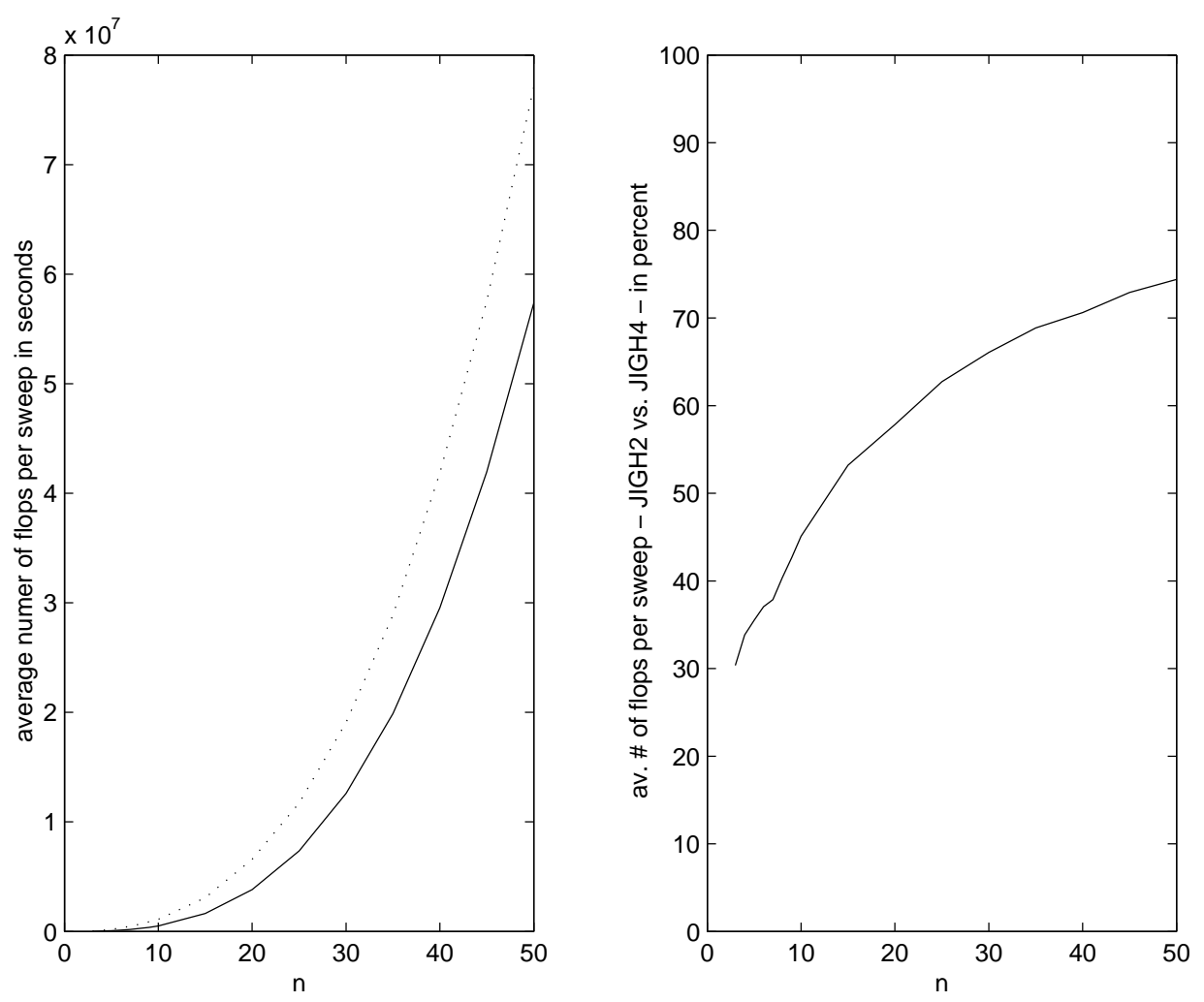

Figure 3: flop count, '-' JIGH2, '. '’' JIGH4

The right graph of Figure 3 displays the average flop count per sweep for JIGH2 in percentage of the one for JIGH4. Clearly, the corresponding flop count for MJIGH2 is somewhere in between the ones for JIGH2 and JIGH4, due to the fact that some loops of MJIGH2 corresponds to loops as in JIGH4 if $2 \times 2$ Hermitian subpencils having real eigenvalues occur. However, the numerical experiments showed that this only happens a few number of times and only during the first few sweeps such that the average flop count per sweep for MJIGH2 is almost the same as the one for JIGH2.

In order to compare the performance of JIGH4 with the one of the algorithm of BunseGerstner/Faßbender for Hamiltonian matrices, a second test was run over 50 pencils of the form

$$
\lambda \mathcal{G}-\mathcal{H}=\left[\begin{array}{cc}
Z_{n} & 0 \\
0 & I_{n}
\end{array}\right]\left(i \lambda J_{n}-J_{n} \mathcal{H}\right)\left[\begin{array}{cc}
Z_{n} & 0 \\
0 & I_{n}
\end{array}\right]
$$

with $\operatorname{norm}(\mathcal{G}, \mathcal{H})=1$, where $Z_{n}$ is the $n \times n$ matrix with ones on the anti-diagonal and zeros elsewhere and $\mathcal{H} \in \mathbb{C}^{2 n \times 2 n}$ is a randomly generated Hamiltonian matrix having no eigenvalues on the imaginary axis. (Such matrices have been used for the numerical experiments in [2].) Thus, pencils as in (25) can be interpreted as 'Hamiltonian matrix'pencils and we expect JIGH4 to perform similarly for them as the method of BunseGerstner/Faßbender does for Hamiltonian matrices. 

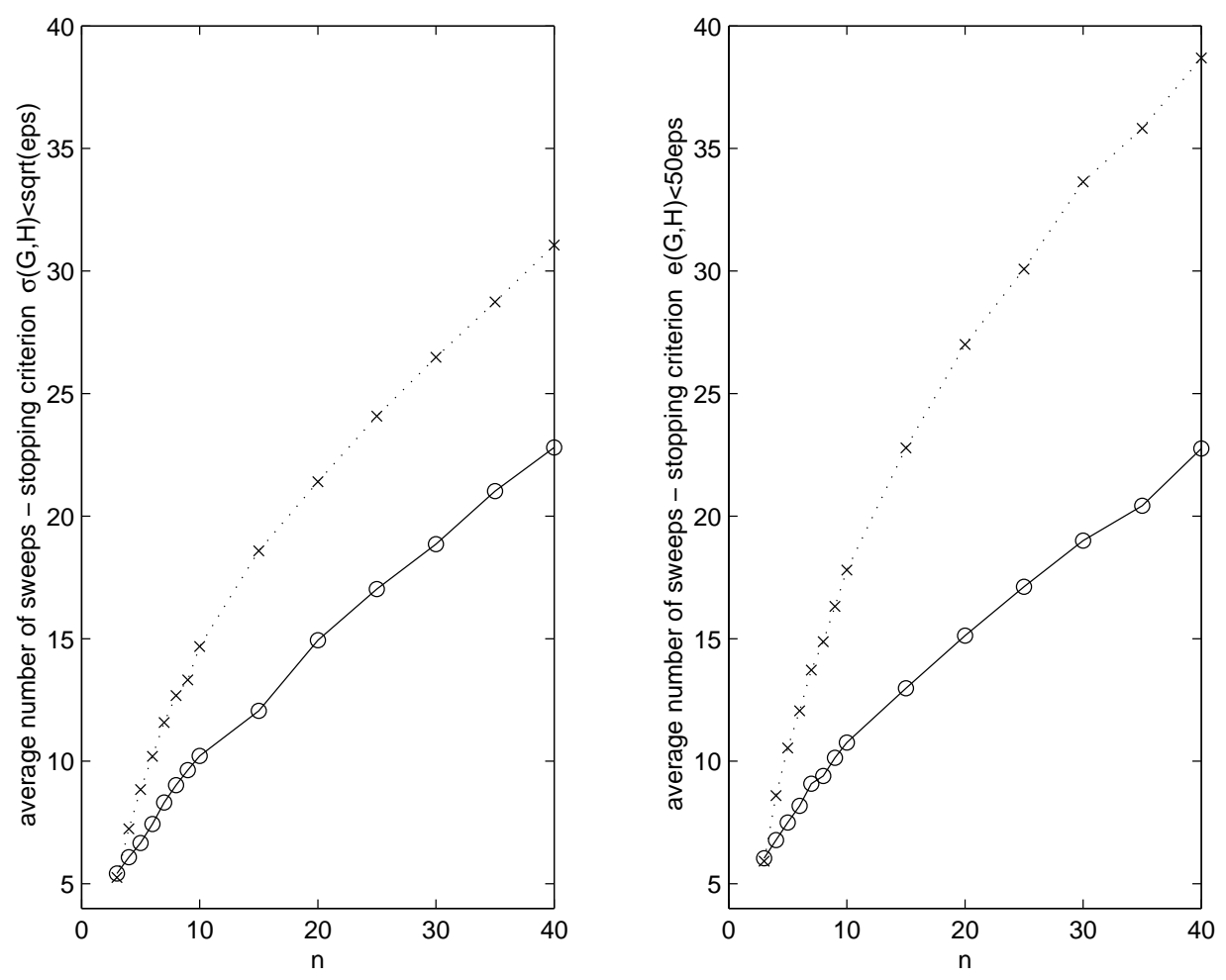

Figure 4: 50 random 'Hamiltonian matrix'-pencils, '_-' MJIGH2, '...' JIGH4

Figure 4 shows the average number of sweeps necessary for convergence for different dimensions $n=3,4, \ldots, 9,10,15,20,25,30,35,40$. For the left graph, we used a stopping criterion corresponding to the one used in [2], i.e., we chose the criterion

$$
\sigma(\mathcal{G}, \mathcal{H}):=\sqrt{\sum_{i=1}^{2 n-1} \sum_{j=1}^{i}\left(\left\|g_{j, i+1-j}\right\|^{2}+\left\|h_{j, i+1-j}\right\|^{2}\right)}<\sqrt{\mathrm{eps}}
$$

Indeed, the graph corresponding to the algorithm JIGH4 is quite similar to the graph obtained by Bunse-Gerstner/Faßbender for their experiments on Hamiltonian matrices. This shows that JIGH4 performs indeed similar to the algorithm by Bunse-Gerstner/Faßbender for Hamiltonian matrices, as it was expected.

On the other hand, the average number of sweeps was always lower with MJIGH2 than with JIGH4. This becomes even more evident in the right graph where we used the more rigid stopping criterion (24). Note that there is hardly any difference of the two graphs corresponding to MJIGH2. Once, the stopping criterion (26) was satisfied with MJIGH2, then at most (if at all) one additional sweep was needed such that the stopping criterion (24) was also satisfied. This is due to the fact that the convergence rate of MJIGH2 was already

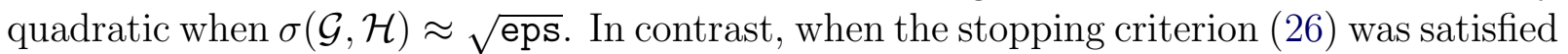
with JIGH4, then several additional sweeps (e.g., an average number of eight sweeps in 
the case $n=40$ ) were needed until the stopping criterion (24) was also satisfied. Note that for both JIGH4 and MJIGH2 the average number of sweeps needed for convergence was always lower for 'Hamiltonian matrix' pencils than for randomly generated Hermitian pencils. This is obviously caused by the rather simple structure of $\mathcal{G}$.

A third test was run over 50 Hermitian pencils close to being in anti-triangular form. For this, we randomly generated Hermitian pencils $\lambda \mathcal{G}-\mathcal{H}$ having no real eigenvalues and with $\operatorname{norm}(\lambda \mathcal{G}-\mathcal{H})=1$. Then, we used JIGH4 to reduce those pencils to anti-triangular form and we randomly generated Hermitian pencils $\lambda \tilde{\mathcal{G}}-\tilde{\mathcal{H}}$ with $\operatorname{norm}(\lambda \tilde{\mathcal{G}}-\tilde{\mathcal{H}})=0.01$. Then the test was run over 50 pencils of the form $\lambda(\mathcal{G}+\tilde{\mathcal{G}})-(\mathcal{H}+\tilde{\mathcal{H}})$, where again we only allowed pencils having no real eigenvalues.

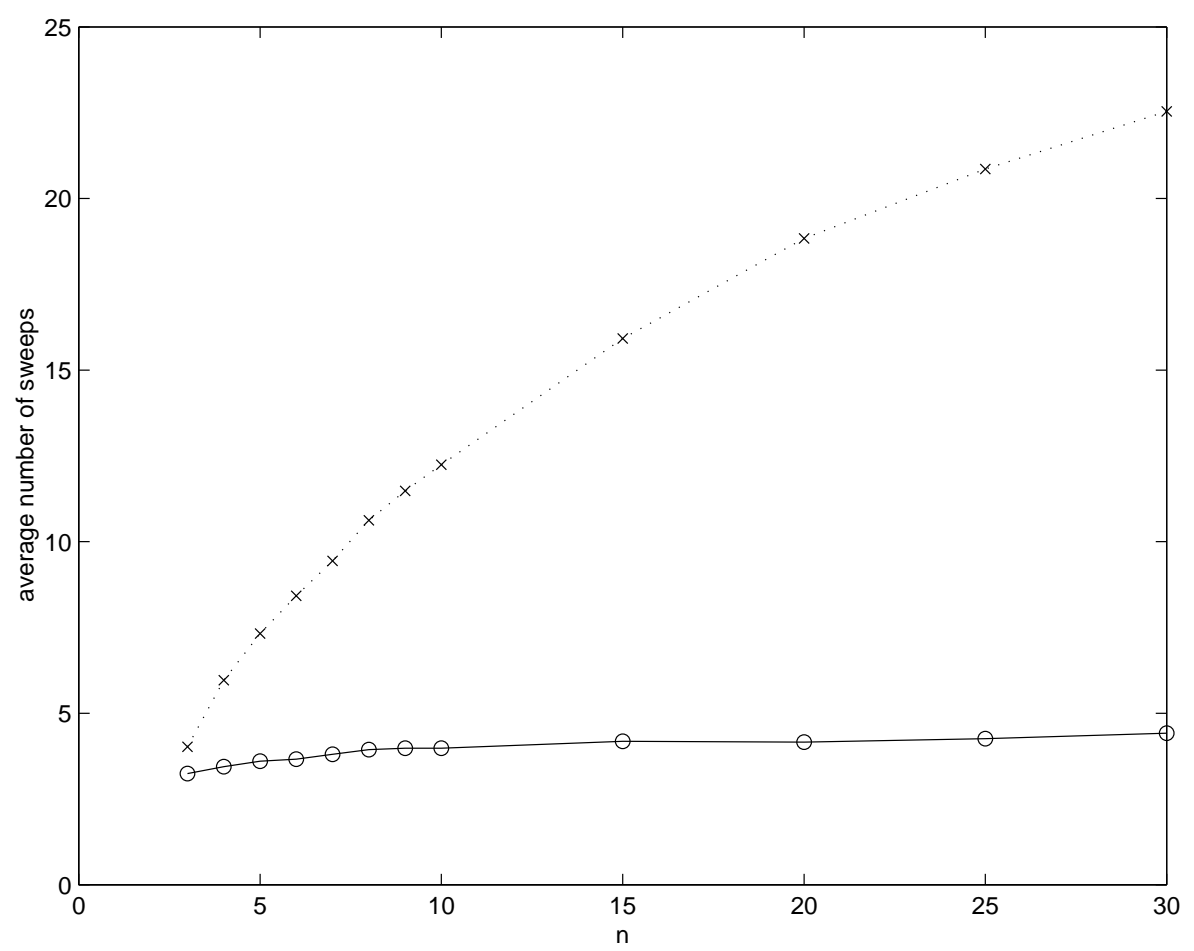

Figure 5: 50 Hermitian pencils close to anti-triangular form, '-' MJIGH2, '‥' JIGH4

Figure 5 shows the average number of sweeps necessary for convergence. Note that this number increases drastically with JIGH4. For the dimension $n=30$ an average of already more than 20 sweeps was necessary for convergence. Although this number is much lower than the corresponding one for randomly generated Hermitian pencils, it is way to high to be favorable considering the fact that the pencil is already close to being in anti-triangular form. On the other hand, the average number of sweeps necessary for convergence was almost constant between 4 and 5 with MJIGH2. Thus, the convergence rate of MJIGH2 was almost quadratic already from the start. Hence, MJIGH2 exploits the special structure of Hermitian pencils that are close to anti-triangular form much better than JIGH4 does. 


\section{Conclusions}

We presented Jacobi-like methods for the solution of the indefinite generalized Hermitian eigenvalue problem. JIGH4 generalizes the method of Bunse-Gerstner/Faßbender for Hamiltonian matrices [2] while MJIGH2 is a slightly modified version of the locally quadratically convergent method JIGH2 that can be interpreted as a direct generalization of Eberlein's method [7] to the case of Hermitian pencils. Numerical experiments show that MJIGH2 is faster and less expensive than JIGH4. Moreover, MJIGH2 excels by the almost constant low number of sweeps needed for convergence if the Hermitian pencil under consideration is already close to being in anti-triangular form. Hence, this algorithm may become attractive for the solution of such generalized Hermitian eigenvalue problems.

Several aspects have not been addressed in this paper. One the one hand, we restricted our research to Hermitian pencils of even size, but we note that a generalization to the case of odd-sized pencils is possible. On the other hand, Bunse-Gerstner/Faßbender showed in [2] that their method allows parallel implementation. Clearly, this is possible for JIGH4 as well, while for MJIGH2 further modifications and investigations are needed.

\section{Acknowledgment}

The author likes to thank V. Mehrmann, K. Veselić, and in particular H. Faßbender for many helpful discussions on this topic.

\section{References}

[1] M. Borri and P. Mantegazza. Efficient solution of quadratic eigenproblems arising in dynamic analysis of structures. Comput. Methods Appl. Mech. Engrg, 12 No.1:19-31, 1977.

[2] A. Bunse-Gerstner and H. Faßbender. A Jacobi-like method for solving algebraic Riccati equations on parallel computers. IEEE Trans. Automat. Control, 42:10711084, 1997.

[3] R. Byers. A Hamiltonian Jacobi algorithm. IEEE Trans. Automat. Control, 35:566570, 1990.

[4] J.P. Charlier and P. van Dooren. A Jacobi-like algorithm for computing the generalized schur form of a regular pencil. J. Comput. Appl. Math., 27:17-36, 1989.

[5] J. Demmel and K. Veselić. Jacobi's method is more accurate than QR. SIAM J. Matrix Anal. Appl., 13:1204-1245, 1992.

[6] P.J. Eberlein. A Jacobi-like method for the automatic computation of eigenvalues and eigenvectors of an arbitrary matrix. J. Soc. Indust. Appl. Math., 10:74-88, 1962. 
[7] P.J. Eberlein. On the schur decomposition of a matrix for parallel computation. IEEE Trans. Comp., 36:167-174, 1987.

[8] H. Faßbender, D.S. Mackey, and N. Mackey. Hamiltonian and Jacobi come full circle: Jacobi algorithms for structured Hamiltonian eigenproblems. Linear Algebra Appl., 332-334:37-80, 2001.

[9] I. Gohberg, P. Lancaster, and L. Rodman. Matrix polynomials. Academic Press, Inc. [Harcourt Brace Jovanovich, Publishers], New York-London, 1982.

[10] H.H. Goldstine and L.P. Horwitz. A procedure for the diagonalization of normal matrices. J. Assoc. Comput. Mach., 6:176-195, 1959.

[11] G.H. Golub and C.F. Van Loan. Matrix Computations. Johns Hopkins University Press, Baltimore, 1983. (third edition: 1996).

[12] J.-S. Guo, W.-W. Lin, and C.S. Wang. Numerical solutions for large sparse quadratic eigenvalue problems. Linear Algebra Appl., 225:57-89, 1995.

[13] D. Hacon. Jacobi's method for skew-symmetric matrices. SIAM J. Matrix Anal. Appl., 14:619-628, 1993.

[14] P. Henrici. On the speed of convergence of cyclic and quasicyclic Jacobi methods for computing the eigenvalues of hermitian matrices. J. Soc. Indust. Appl. Math, 6:144-162, 1958.

[15] C.G.J. Jacobi. Über ein leichtes Verfahren, die in der Theorie der Säcularströmungen vorkommenden Gleichungen numerisch aufzulösen. J. Reine Angew. Math., 30:51-95, 1846.

[16] H.P.M. van Kempen. On the convergence of the classical Jacobi method for real symmetric matrices with non-distinct eigenvalues. Numer. Math., 9:11-18, 1966.

[17] P. Lancaster. Lambda-matrices and vibrating systems. International Series of Monographs in Pure and Applied Mathematics, Vol. 94. Pergamon Press, Oxford-New York-Paris, 1966.

[18] P. Lancaster and L. Rodman. Algebraic Riccati Equations. Clarendon Press, Oxford, 1995.

[19] A.J. Laub. A Schur method for solving algebraic riccati equations. IEEE Trans. Automat. Control, AC-24:913-921, 1979.

[20] R. Mathias. Accurate eigensystem computations by Jacobi methods. SIAM J. Matrix Anal. Appl., 16:977-1003, 1995.

[21] C. Mehl. Condensed forms for skew-Hamiltonian/Hamiltonian pencils. SIAM J. Matrix Anal. Appl., 21:454-476, 1999. 
[22] C. Mehl. Anti-triangular and anti-m-Hessenberg forms for Hermitian matrices and pencils. Linear Algebra Appl., 317:143-176, 2000.

[23] V. Mehrmann. Existence, uniqueness, and stability of solutions to singular linear quadratic optimal control problems. Linear Algebra Appl., 121:291-331, 1989.

[24] V. Mehrmann. The Autonomous Linear Quadratic Control Problem, Theory and Numerical Solution. Number 163 in Lecture Notes in Control and Information Sciences. Springer-Verlag, Heidelberg, July 1991.

[25] M.H.C. Paardekooper. An eigenvalue algorithm for skew-symmetric matrices. Numer. Math., 17:189-202, 1971.

[26] A. Schönhage. Zur quadratischen Konvergenz des Jacobi-Verfahrens. Numer. Math., 6:410-412, 1964.

[27] G.W. Stewart. On the sensitivity of the eigenvalue problem $A x=\lambda B x$. SIAM J. Numer. Anal., 9:669-686, 1972.

[28] G.W. Stewart. A Jacobi-like algorithm for computing the Schur decomposition of a non-Hermitian matrix. SIAM J. Sci. Statist. Comput., 6:853-864, 1985.

[29] G.W. Stewart and J.G. Sun. Matrix perturbation theory. Academic Press, Inc., Boston, 1990.

[30] R.C. Thompson. The characteristic polynomial of a principal subpencil of a Hermitian matrix pencil. Linear Algebra Appl., 14:135-177, 1976.

[31] F. Tisseur. Stability of structured Hamiltonian eigensolvers. SIAM J. Matrix Anal. Appl., 23:103-125, 2001.

[32] F. Tisseur and K. Meerbergen. The quadratic eigenvalue problem. SIAM Rev., 43:235$286,2001$.

[33] J.H. Wilkinson. Note on the quadratic convergence of the cyclic Jacobi process. Numer. Math, 4:296-300, 1962.

[34] K. Zhou, J.C. Doyle, and K. Glover. Robust and optimal control. Prentice-Hall, Upper Saddle River, NJ, 1995. 